TRANSACTIONS OF THE

AMERICAN MATHEMATICAL SOCIETY

Volume 354, Number 7 , Pages 2639-2658

S 0002-9947(02)02953-7

Article electronically published on February 4, 2002

\title{
ON FANO MANIFOLDS WITH NEF TANGENT BUNDLES ADMITTING 1-DIMENSIONAL VARIETIES OF MINIMAL RATIONAL TANGENTS
}

\author{
NGAIMING MOK
}

\begin{abstract}
Let $X$ be a Fano manifold of Picard number 1 with numerically effective tangent bundle. According to the principal case of a conjecture of Campana-Peternell's, $X$ should be biholomorphic to a rational homogeneous manifold $G / P$, where $G$ is a simple Lie group, and $P \subset G$ is a maximal parabolic subgroup.

In our opinion there is no overriding evidence for the Campana-Peternell Conjecture for the case of Picard number 1 to be valid in its full generality. As part of a general programme that the author has undertaken with Jun-Muk Hwang to study uniruled projective manifolds via their varieties of minimal rational tangents, a new geometric approach is adopted in the current article in a special case, consisting of (a) recovering the generic variety of minimal rational tangents $\mathcal{C}_{x}$, and (b) recovering the structure of a rational homogeneous manifold from $\mathcal{C}_{x}$. The author proves that, when $b_{4}(X)=1$ and the generic variety of minimal rational tangents is 1-dimensional, $X$ is biholomorphic to the projective plane $\mathbb{P}^{2}$, the 3 -dimensional hyperquadric $Q^{3}$, or the 5 -dimensional Fano homogeneous contact manifold of type $G_{2}$, to be denoted by $K\left(G_{2}\right)$.

The principal difficulty is part (a) of the scheme. We prove that $\mathcal{C}_{x} \subset$ $\mathbb{P} T_{x}(X)$ is a rational curve of degrees $\leq 3$, and show that $d=1$ resp. 2 resp. 3 corresponds precisely to the cases of $X=\mathbb{P}^{2}$ resp. $Q^{3}$ resp. $K\left(G_{2}\right)$. Let $\mathcal{K}$ be the normalization of a choice of a Chow component of minimal rational curves on $X$. Nefness of the tangent bundle implies that $\mathcal{K}$ is smooth. Furthermore, it implies that at any point $x \in X$, the normalization $\mathcal{K}_{x}$ of the corresponding Chow space of minimal rational curves marked at $x$ is smooth. After proving that $\mathcal{K}_{x}$ is a rational curve, our principal object of study is the universal family $\mathcal{U}$ of $\mathcal{K}$, giving a double fibration $\rho: \mathcal{U} \rightarrow \mathcal{K}, \mu: \mathcal{U} \rightarrow X$, which gives $\mathbb{P}^{1}$-bundles. There is a rank-2 holomorphic vector bundle $V$ on $\mathcal{K}$ whose projectivization is isomorphic to $\rho: \mathcal{U} \rightarrow \mathcal{K}$. We prove that $V$ is stable, and deduce the inequality $d \leq 4$ from the inequality $c_{1}^{2}(V) \leq 4 c_{2}(V)$ resulting from stability and the existence theorem on Hermitian-Einstein metrics. The case of $d=4$ is ruled out by studying the structure of the curvature tensor of the Hermitian-Einstein metric on $V$ in the special case where $c_{1}^{2}(V)=4 c_{2}(V)$.
\end{abstract}

According to Mori's solution to Hartshorne's Conjecture ([Mr], 1979), a projective manifold with ample tangent bundle is isomorphic to the projective space $\mathbb{P}^{n}$. In Kähler geometry, Siu and Yau ([SY], 1980) solved the Frankel Conjecture by differential-geometric means, showing that a compact Kähler manifold with positive bisectional curvature is biholomorphic to the projective space. The author solved in $(\underline{\mathrm{Mk}}, 1988)$ the Generalized Frankel Conjecture, affirming in particular

Received by the editors December 31, 2000.

2000 Mathematics Subject Classification. Primary 14J60, 53C07.

(C)2002 American Mathematical Society 
that a compact Kähler manifold of semipositive holomorphic bisectional curvature and positive Ricci curvature is necessarily biholomorphic to a Hermitian symmetric space of the compact type. In view of this, Campana and Peternell ([CP1], 1991) formulated a conjectural algebro-geometric analogue, according to which a Fano manifold $X$ with nef tangent bundle should be isomorphic to a rational homogeneous manifold $G / P$, where $G$ is a semisimple Lie group and $P \subset G$ is a parabolic subgroup. If we impose the additional condition that $X$ is of Picard number 1, which is the crucial case for Fano manifolds, $G$ should be simple and $P \subset G$ should be a maximal parabolic subgroup.

In a series of articles, Jun-Muk Hwang and the author have been studying Fano manifolds via a geometric study of moduli spaces of minimal rational curves and their associated varieties of minimal tangents (cf. [HM3] for a recent survey). For Fano manifolds with nef tangent bundle, there is no obstruction to the deformation of rational curves (fixing a base point). As a consequence, the universal family associated to an irreducible component $\mathcal{K}$ of minimal rational curves leads to a double fibration over the base manifold and over $\mathcal{K}$ (which will also be called a minimal rational component). One hope for recovering structures of rational homogeneous manifolds from nefness of the tangent bundle is via the study of the double fibration. From this approach the complexity of the conjecture is directly related to the dimension of varieties of minimal rational tangents, without any $a$ priori assumption on the dimension of the base manifold $X$ itself. In this article we treat a particular case, by imposing the condition that varieties of minimal rational tangents are 1-dimensional. With one additional topological condition that the fourth Betti number $b_{4}(X)=1$, we show that $X$ must be isomorphic to one of the 3 known possibilities: the projective plane $\mathbb{P}^{2}$, the 3 -dimensional hyperquadric $Q^{3}$, and the 5-dimensional Fano homogeneous contact manifold associated to the simple Lie group $G_{2}$.

The key to the proof of our theorem is an a priori bound on the degree of varieties of minimal rational tangents. With some oversimplification our proof proceeds as follows. By restricting to minimal rational curves it follows that normalized spaces of minimal rational curves marked at an arbitrary point are projective-algebraic curves of genus 0 . The double fibration arising from the universal family of minimal rational curves gives rise to a holomorphic $\mathbb{P}^{1}$-bundle, which is the projectivization of a rank-2 holomorphic vector bundle $V$ over the minimal rational component $\mathcal{K}$. We prove that $V$ is stable, implying an inequality involving first and second Chern classes as a consequence of the existence of Hermitian-Einstein metrics. From this and the assumption that $b_{4}(X)=1$ we deduce that generic varieties of minimal rational tangents are of degree $\leq 3$, and proceed to prove that they correspond to the 3 known possibilities of rational homogeneous manifolds of Picard number 1 carrying 1-dimensional varieties of minimal rational tangents, as listed above.

At this point there is in our opinion no convincing evidence why the CampanaPeternell Conjecture should be valid in its full generality. It is therefore interesting to seek supplementary geometric hypotheses under which special cases of the conjecture can be ascertained. The present article represents a first result in the direction of recovering global "symmetry" (in the sense of symmetry under group actions) by means of a determination of generic varieties of minimal rational curves. It is also the first instance where the structure of some non-symmetric rational homogeneous manifold of Picard number 1 can be reconstructed from the nefness assumption. In view of the scarcity of results in relation to the conjecture, especially in view of the 
absence of results in which there are no a priori restrictions on the dimension of the ambient manifold, we deem it worthwhile to present the partial results of the current article to provide a new perspective on the conjecture.

\section{StATEMENT OF RESUlts AND BACKGROUND ON UNIVERSAL FAMILIES}

(1.1). Let $X$ be a Fano manifold with nef tangent bundle. Campana and Peternell [CP1] conjectured that $X$ is biholomorphic to a rational homogeneous manifold. Using the classification theory of Fano manifolds, they solved the conjecture for dimensions up to 3. The same result was obtained by Zheng [Zh]. For dimension 4 Campana and Peternell [CP2] solved the conjecture with one possible exception. There a principal ingredient is Fujita's classification theory of polarized varieties $\mathrm{Fu}$. or alternatively the classification of Fano manifolds of co-index 3 according to Mukai $[\mathrm{Mu}]$. For the case of Fano manifolds of Picard number 1 results on the Conjecture can be more precisely stated as follows.

Theorem (1.1.1) (from Campana-Peternell [CP1], [CP2], Zheng [Zh]). Let X be a Fano manifold with nef tangent bundle and of Picard number 1, of dimension $\leq 4$. Then $X$ must be biholomorphic to a projective space $\mathbb{P}^{n}, 1 \leq n \leq 4$, a hyperquadric $Q^{n}, n=3,4$, or a 4-dimensional Fano manifold whose first Chern class is a generator of $H^{2}(X, \mathbb{Z}) \cong \mathbb{Z}$.

It is expected that the last alternative does not actually occur.

In a series of articles, Jun-Muk Hwang and the author have been studying Fano manifolds via their varieties of minimal rational tangents (VMRTs). In this article the author studies a particular case of the Campana-Peternell Conjecture from this perspective, where a generic VMRT is 1-dimensional, without any assumption on the dimension of $X$. For Fano manifolds with nef tangent bundle, there is no obstruction to the deformation of rational curves (fixing a base point), and the universal family associated to a minimal rational component $\mathcal{K}$ leads to a double fibration $\rho: \mathcal{U} \longrightarrow \mathcal{K}, \mu: \mathcal{U} \longrightarrow X$. In the case where a generic VMRT is 1-dimensional, it is a rational curve, and the double fibration gives two holomorphic $\mathbb{P}^{1}$-bundles. The main input of the article is an a priori bound $\operatorname{deg}\left(\mathcal{C}_{x}\right) \leq 3$ for the degree of a generic VMRT $\mathcal{C}_{x}$ under the topological assumptions $b_{2}(X)=b_{4}(X)=1$. The bound $\operatorname{deg}\left(\mathcal{C}_{x}\right) \leq 3$ is obtained from the stability of $V$ and Chern-class inequalities resulting from the existence of Hermitian-Einstein metrics. Finally, using arguments involving distributions spanned by VMRTs and geometric structures, we recover the rational homogeneous structure from 1-dimensional VMRTs of degrees $\leq 3$. Our main result is

Main Theorem. Let $X$ be a Fano manifold with nef tangent bundle satisfying $b_{2}(X)=b_{4}(X)=1$. Suppose $X$ carries a rational curve $C$ such that $K_{X}^{-1} \cdot C=3$. Then, $X$ is biholomorphic to one of the following distinct rational homogeneous manifolds:

(a) the projective plane $\mathbb{P}^{2}$;

(b) the 3-dimensional hyperquadric $Q^{3}$;

(c) the 5-dimensional Fano homogeneous contact manifold of type $G_{2}$.

Geometrically the hypothesis $K_{X}^{-1} \cdot C=3$ means that the generic VMRT $\mathcal{C}_{x}$ is 1-dimensional. 
(1.2). Let $X$ be a uniruled projective manifold. Fixing a polarization, we have the notion of minimal rational curves (cf. Hwang-Mok [HM3, §1]). Let $\mathcal{K}^{o}$ be a normalized irreducible component of minimal rational curves, and $\mathcal{K}$ an irreducible normal projective variety of 1-cycles on $X$ containing $\mathcal{K}^{o}$ as a Zariski-dense open subset. We call $\mathcal{K}$ a minimal rational component. The starting point of our approach is a study of the universal family associated to a minimal rational component $\mathcal{K}$ on a Fano manifold $X$ with nef tangent bundle. We have, first of all,

Lemma (1.2.1). Let $X$ be a uniruled projective manifold with nef tangent bundle, and let $\mathcal{K}$ be a minimal rational component on $X$, with respect to a polarization on $X$. Then, every member of $\mathcal{K}$ is a generically reduced free minimal rational curve, and $\mathcal{K}$ is a projective manifold of dimension $n+\ell-3$, where $\ell=K_{X}^{-1} \cdot C$ for any $[C] \in \mathcal{K}$. Furthermore, the universal family $\rho: \mathcal{U} \longrightarrow \mathcal{K}$ is a holomorphic $\mathbb{P}^{1}$-bundle.

Proof. Let $f: \mathbb{P}^{1} \longrightarrow X$ be any non-trivial holomorphic map. Since $X$ has nef tangent bundle, $f^{*} T_{X}$ is nef on $\mathbb{P}^{1}$, so that $f^{*} T_{X} \equiv \mathcal{O}\left(a_{1}\right) \oplus \cdots \oplus \mathcal{O}\left(a_{n}\right)$ with $a_{1}, \cdots, a_{n} \geq 0$. Thus, $f$ is free and there is no obstruction to local deformations of $f$. As a consequence, the space of maps $\operatorname{Hom}\left(\mathbb{P}^{1} ; X\right)$ is smooth at $[f]$, of complex dimension $\operatorname{dim} H^{0}\left(\mathbb{P}^{1}, f^{*} T_{X}\right)=n+\ell$. We assert that for any minimal rational component $\mathcal{K}$, any $[C] \in \mathcal{K}$ must be irreducible and generically reduced. Otherwise one can always take the reduction $C^{\prime}$ of any irreducible component of $C$, with a parametrization $g: \mathbb{P}^{1} \longrightarrow C^{\prime} \subset X$. Then, $g^{*} T_{X}$ is nef and $C^{\prime}$ is free, of degree $<\operatorname{deg}(C)$, contradicting minimality. From the irreducibility of every $[C] \in \mathcal{K}$ it follows that $\mathcal{K}=\operatorname{Hom}_{0}\left(\mathbb{P}^{1} ; X\right) / A u t\left(\mathbb{P}^{1}\right)$ for some irreducible component $\operatorname{Hom}_{0}\left(\mathbb{P}^{1} ; X\right)$ of $\operatorname{Hom}\left(\mathbb{P}^{1} ; X\right)$. As a consequence, $\mathcal{K}$ is smooth, projective and of dimension $n+\ell-3$. The universal family is constructed from the canonical $\operatorname{map} \mathcal{U}:=\operatorname{Hom}_{0}\left(\mathbb{P}^{1} ; X\right) / A u t\left(\mathbb{P}^{1} ; 0\right) \longrightarrow \operatorname{Hom}_{0}\left(\mathbb{P}^{1} ; X\right) / A u t\left(\mathbb{P}^{1}\right)=\mathcal{K}$, with fibers $\cong \operatorname{Aut}\left(\mathbb{P}^{1}\right) / A u t\left(\mathbb{P}^{1} ; 0\right) \cong \mathbb{P}^{1}$, defining a structure of a holomorphic $\mathbb{P}^{1}$-bundle for $\rho: \mathcal{U} \longrightarrow \mathcal{K}$.

$\mathcal{U}$ is also equipped with a canonical projection $\mu: \mathcal{U} \rightarrow X$, defined by $\mu([f])=$ $f(0) . \rho: \mathcal{U} \rightarrow \mathcal{K}, \mu: \mathcal{U} \rightarrow X$ gives the universal family associated to $\mathcal{K}$. For $x \in X$ let $\operatorname{Hom}_{0}\left(\left(\mathbb{P}^{1}, 0\right) ;(X, x)\right) \subset \operatorname{Hom}_{0}\left(\left(\mathbb{P}^{1}, X\right)\right.$ be the subset consisting of $f: \mathbb{P}^{1} \rightarrow X$ satisfying $f(0)=x$. We call $\mathcal{U}_{x}:=\operatorname{Hom}_{0}\left(\left(\mathbb{P}^{1}, 0\right) ;(X, x)\right) / \operatorname{Aut}\left(\mathbb{P}^{1}, 0\right)$ the moduli space of marked $\mathcal{K}$-rational curves at $x$, which is the same as the fiber $\mu^{-1}(x)$ of $\mu: \mathcal{U} \rightarrow X$. For $X$ with nef tangent bundle we have

Lemma (1.2.2). Let $X$ be a uniruled projective manifold with nef tangent bundle, and let $\mathcal{K}$ be a minimal rational component on $X$. Then, the normalization $\mathcal{U}_{x}$ of the moduli space of marked $\mathcal{K}$-rational curves at any point $x$ is a connected projective manifold, and the canonical map $\mu: \mathcal{U} \longrightarrow X$ is a holomorphic submersion. Furtheremore, if the $V M R T \mathcal{C}_{x}$ is 1-dimensional at a generic point, every fiber $\mathcal{U}_{x}$ of $\mu: \mathcal{U} \longrightarrow X$ is a projective curve of genus 0 .

Proof. Let $x \in X$ be an arbitrary base point and $C$ be any minimal rational curve on $X$ passing through $x$ and belonging to $\mathcal{K}$, represented by $f: \mathbb{P}^{1} \longrightarrow X$ with $f(0)=$ $x$. By Lemma (1.2.1), $C=f\left(\mathbb{P}^{1}\right)$ is free. Furthermore, since $a_{i}-1 \geq-1$ for the degrees $a_{i}$ of the Grothendieck components of $f^{*} T_{X}$, we have $H^{1}\left(\mathbb{P}^{1}, f^{*} T_{X} \otimes \mathfrak{m}_{0}\right)=$ 0 , where $\mathfrak{m}_{0}$ stands for the maximal ideal sheaf defined by $0 \in \mathbb{P}^{1}$, so that there is no obstruction to local deformations $f_{t}$ of $f: \mathbb{P}^{1} \longrightarrow X$ with $f_{t}(0)=f(0)=x$. It 
follows that $\mathcal{U}_{x}$ is smooth, and that $\mu: \mathcal{U} \longrightarrow X$ is a holomorphic submersion (cf. Hwang-Mok [HM2, Proposition 4]). Since $\mathcal{K}$ and hence $\mathcal{U}$ is connected, $\mu: \mathcal{U} \longrightarrow X$ is a regular family, and the Fano manifold $X$ is simply-connected, all fibers $\mathcal{U}_{x}$ must be connected.

If now the VMRT $\mathcal{C}_{x}$ is 1 -dimensional at a generic point $x \in X$, then $\mathcal{U}_{x}$ is 1-dimensional for every point $x \in X$. Let $C$ be a minimal rational curve belonging to $\mathcal{K}$ and consider the regular family $\left.\mu\right|_{C}:\left.\mathcal{U}\right|_{C} \longrightarrow C$ of compact Riemann surfaces over $C$. Let $f: \mathbb{P}^{1} \longrightarrow C$ be the normalization of $C$ and $f^{*} \mu: f^{*} \mathcal{U} \longrightarrow \mathbb{P}^{1}$ the corresponding regular family over $\mathbb{P}^{1}$. If the fibers $\mathcal{U}_{x}$ are of genus $>0$, then the regular family over $\mathbb{P}^{1}$ must be trivial. On the other hand, consider the tautological lifting $\hat{C}$ of $C$ to $\left.\mathcal{U}\right|_{C}$, which corresponds to a section $S$ of $f^{*} \mu: f^{*} \mathcal{U} \longrightarrow \mathbb{P}^{1}$ over $\mathbb{P}^{1}$. The map $f^{*} \rho: f^{*} \mathcal{U} \longrightarrow \mathcal{K}$ collapses $S$ to a point $[C] \in \mathcal{K}$ but is of maximal rank at a generic point of $f^{*} \mathcal{U}$. It follows that $S \subset f^{*} \mathcal{U}$ is an exceptional curve, contradicting the triviality of $f^{*} \mu: f^{*} \mathcal{U} \longrightarrow \mathbb{P}^{1}$ under the hypothesis that fibers are of genus $>0$. We have thus proven that all fibers of $\mu: \mathcal{U} \longrightarrow X$ are of genus 0 , as desired.

Remarks. Although $\mu: \mathcal{U} \longrightarrow X$ is a regular family, the image of a fiber $\mathcal{U}_{x}$ under $\rho: \mathcal{U} \longrightarrow \mathcal{K}$ may be singular. This is the case at a point $z \in \mathcal{U}_{x}$ corresponding to a locally irreducible branch of some $[C] \in \mathcal{K}$ singular at the point $x$.

We will construct a rank- 2 holomorphic vector bundle $V$ on $\mathcal{K}$ whose projectivization is isomorphic to $\rho: \mathcal{U} \longrightarrow \mathcal{K}$. On the minimal rational component $\mathcal{K}$, for the purpose of computing Chern classes, we need to relate cohomological invariants of $\mathcal{K}$ to those of $X$, using the double fibration given by the universal family. We have, first of all,

Lemma (1.2.3). Let $X$ be a Fano manifold with nef tangent bundle satisfying the hypothesis of the Main Theorem. Let $\mathcal{K}$ be the minimal rational component on $X$ such that $[C] \in \mathcal{K}$. Then, $\mathcal{K}$ is smooth and $b_{2}(\mathcal{K})=b_{4}(\mathcal{K})=1$.

Proof. Let $\mu: \mathcal{U} \longrightarrow X, \rho: \mathcal{U} \longrightarrow \mathcal{K}$ be the universal family associated to $\mathcal{K}$. Since $X$ has nef tangent bundle, every rational curve on $X$ is free. It follows in particular that $\mathcal{K}$ is smooth, and that $\rho: \mathcal{U} \longrightarrow K$ is a holomorphic $\mathbb{P}^{1}$-bundle. By Lemma (1.2.2), $\mu: \mathcal{U} \longrightarrow X$ is also a holomorphic $\mathbb{P}^{1}$-bundle. By the Gysin sequence for sphere bundles (cf. Spanier [Sp p.499, Theorem 2]) we have, for any integer $k$,

$$
\begin{gathered}
b_{k+2}(\mathcal{U})=b_{k}(X)+b_{k+2}(X), \\
b_{k+2}(\mathcal{U})=b_{k}(\mathcal{K})+b_{k+2}(\mathcal{K}),
\end{gathered}
$$

where $b_{k}=0$ for $k<0$. It follows that $b_{k}(X)=b_{k}(\mathcal{K})$ for any $k$. In particular $b_{2}(\mathcal{K})=b_{4}(\mathcal{K})=1$, as desired.

(1.3). Using results of (1.2), we consider the question of constructing a rank-2 holomorphic vector bundle whose projectivization is the holomorphic $\mathbb{P}^{1}$-bundles $\rho: \mathcal{U} \rightarrow \mathcal{K}$.

Lemma (1.3.1). Let $M$ be a complex manifold and $\pi: \mathcal{P} \longrightarrow M$ a holomorphic $\mathbb{P}^{1}$-bundle. Suppose there exists a holomorphic line bundle $E$ on $\mathcal{P}$ such that $\left.E\right|_{\pi^{-1}(m)} \cong \mathcal{O}(1)$ for $m \in M$, where $\mathcal{O}(1)$ denotes the positive generator of the Picard group of $\pi^{-1}(m) \cong \mathbb{P}^{1}$. Then, $\mathcal{P}$ is isomorphic to the projectivization $\mathbb{P} V$ of some rank-2 holomorphic vector bundle $V$ over $M$. 
Proof. Let $\mathcal{E}$ be the locally free sheaf on $\mathcal{P}$ corresponding to $E$, and let $\mathcal{V}=\pi_{*} \mathcal{E}$ be its direct image on $M$. Since $\left.E\right|_{\pi^{-1}(m)} \cong \mathcal{O}(1), \mathcal{V}$ is locally free, of rank = $\operatorname{dim} \Gamma\left(\pi^{-1}(m), \mathcal{O}(1)\right)=2$. Let $\lambda: V \rightarrow M$ be the rank-2 holomorphic vector bundle corresponding to $\mathcal{V}$. For $m \in M$ write $V_{m}$ for the fiber $\lambda^{-1}(m)$. To each $p \in \mathcal{P}_{m}:=\pi^{-1}(m)$ we associate the 1-dimensional vector subspace $J_{p}:=\left\{\sigma \in V_{m}\right.$ : $\sigma(p)=0\} \subset V_{m}^{*}$. The mapping $p \mapsto\left[J_{p}\right] \in \mathbb{P} V^{*}$ then defines an isomorphism from $\mathcal{P}$ to $\mathbb{P} V^{*}$ as $\mathbb{P}^{1}$-bundles. For the rank-2 holomorphic vector bundle $V$ we have $V \cong V^{*} \otimes \operatorname{det}(V)$, so that $\mathbb{P} V \cong \mathbb{P} V^{*}$, giving $\mathbb{P} V \cong \mathcal{P}$, as desired.

Corollary (1.3.1). Let $X$ be a Fano manifold with nef tangent bundle satisfying the hypothesis of the Main Theorem. Let $\rho: \mathcal{U} \longrightarrow \mathcal{K}$ be the universal family associated to a choice of minimal rational component $\mathcal{K}$ on $X$. Then, there exists a rank-2 holomorphic vector bundle $\lambda: V \rightarrow \mathcal{K}$ such that the $\mathbb{P}^{1}$-bundle $\rho: \mathcal{U} \longrightarrow \mathcal{K}$ is isomorphic to the projectivization $[\lambda]: \mathbb{P} V \longrightarrow \mathcal{K}$ as holomorphic $\mathbb{P}^{1}$-bundles.

Proof. By Lemma (1.3.1) it suffices to show that there exists a holomorphic line bundle $E$ on $\mathcal{U}$ such that $\left.E\right|_{\hat{C}} \cong \mathcal{O}(1)$. The minimal rational curve $C \subset X$ satisfies $K_{X}^{-1} \cdot C=3$, so that $C$ is of degree 1 or 3 . If $C$ is of degree 1 , then $E=\mu^{*}(\mathcal{O}(1))$ satisfies the property desired, where $\mathcal{O}(1)$ denotes the positive generator of $\operatorname{Pic}(X)$. If $C$ is of degree 3 , then $F:=\left.\mu^{*}(\mathcal{O}(1))\right|_{\hat{C}} \cong \mathcal{O}(3)$. On the other hand, for the tautological line bundle $L$ on $\mathbb{P} T_{X}, \tau^{*} L$ defines a holomorphic line bundle $H$ on $\mathcal{U}-\mathcal{Z}$ for some subvariety $\mathcal{Z} \subset \mathcal{U}$ of codimension $\geq 2$, such that the corresponding locally free sheaf extends to $\mathcal{U}$ as a torsion-free sheaf $\mathcal{H}$. The double dual $\mathcal{H}^{* *}$ is then locally free, thus defining an extension of $H$ to $\mathcal{U}$ as a holomorphic line bundle, still to be denoted by $H$. Denote by $C^{\prime} \subset \mathbb{P} T_{X}$ the tautological lifting of $C$ to $\mathbb{P} T_{X}$. If we choose $C$ to be a standard rational curve, and assume $C^{\prime} \subset \mathbb{P} T_{X}$ to be embedded for notational simplicity, then the tangent map $\tau$ is a holomorphic embedding in a neighborhood of $\hat{C}$, and we can identify $\left.H\right|_{\hat{C}}$ with $\left.L\right|_{C^{\prime}}$. But the latter is isomorphic to $T_{C^{\prime}} \cong \mathcal{O}(2)$. As a consequence, $E:=F \otimes H^{-1}$ gives a holomorphic line bundle on $\mathcal{U}$ such that $\left.E\right|_{\hat{C}} \cong \mathcal{O}(1)$, as desired.

\section{Bounding THE DEGREe OF GENERIC VMRTS USING THE STABILITY OF A RANK-2 BUNDLE}

(2.1). Let $X$ be a Fano manifold with nef tangent bundle satisfying the hypothesis of the Main Theorem. We study the rank-2 holomorphic vector bundles $V$ on $\mathcal{K}$ resp. $X$ given in Corollary (1.3.1). In order to prove the Main Theorem, our approach is first of all to bound the degree of generic VMRTs of $X$. We do this by proving the stability of $V$ and resorting to Chern-class inequalities for stable vector bundles. To start with we need the following elementary lemma on exceptional curves on ruled surfaces over $\mathbb{P}^{1}$.

Lemma (2.1.1). For an integer $c$, denote by $\mathcal{O}(c)$ the holomorphic line bundle of degree $c$ on $\mathbb{P}^{1}$. Let $a>b$ be integers. Then, $\mathbb{P}(\mathcal{O}(a))$ is the unique holomorphic section of $S:=\mathbb{P}(\mathcal{O}(a) \oplus \mathcal{O}(b))$ over $\mathbb{P}^{1}$ which is at the same time an exceptional curve on $S$.

Here and henceforth, by abuse of language we sometimes call "the graph of a holomorphic section" simply "a holomorphic section".

Proof. The canonical projection $\pi: S \longrightarrow \mathbb{P}^{1}$ is a holomorphic $\mathbb{P}^{1}$-bundle. Let

$E$ be the graph of a holomorphic section of $\mathbb{P}(\mathcal{O}(a) \oplus \mathcal{O}(b))$ such that $E$ is an 
exceptional curve on $S$. Then, taking $E$ to be the infinity section of $\pi: S \longrightarrow \mathbb{P}^{1}$, $\left.\pi\right|_{S-E}: S-E \longrightarrow \mathbb{P}^{1}$ is an affine holomorphic $\mathbb{C}$-bundle over $\mathbb{P}^{1}$, to be denoted by $A$. The associated holomorphic line bundle $L$ over $\mathbb{P}^{1}$ is defined by a 1 -cocycle which is the inverse of a 1-cocycle defining the normal bundle $N$ of $E$ in $S$, when $N$ is considered as a holomorphic line bundle over $\mathbb{P}^{1}$ via the isomorphism $\left.\pi\right|_{E}: E \cong \mathbb{P}^{1}$. Since $E \subset S$ is an exceptional curve, $L$ is a positive holomorphic line bundle over $\mathbb{P}^{1}$. As a consequence, $H^{1}\left(\mathbb{P}^{1}, L\right)=0$, and the affine $\mathbb{C}$-bundle $A$ over $\mathbb{P}^{1}$ admits a holomorphic section, whose graph will be denoted by $E_{2}$. It follows that $S$ is isomorphic to $\mathbb{P}\left(\Lambda_{1} \oplus \Lambda_{2}\right)$, where $\Lambda_{1}, \Lambda_{2} \subset \mathcal{O}(a) \oplus \mathcal{O}(b)$ are holomorphic line subbundles, and $E_{1}=\mathbb{P} \Lambda_{1}, E_{2}=\mathbb{P} \Lambda_{2}, E_{1}:=E$. Thus, $\Lambda_{1} \oplus \Lambda_{2} \cong \mathcal{O}(a) \oplus \mathcal{O}(b)$. From the uniqueness of Grothendieck decompositions and the fact that $E_{1}$ is an exceptional curve on $S$ it follows that $\Lambda_{1} \cong \mathcal{O}(a)$ and $\Lambda_{2} \cong \mathcal{O}(b)$. Furtheremore, the direct summands of highest degree $E_{1}$ and $\mathcal{O}(a)$ are actually equal. In other words, $\Lambda_{1}$ is nothing other than $\mathcal{O}(a)$, proving uniqueness of the latter as a holomorphic section which is at the same time an exceptional curve on $S$.

Proposition (2.1.1). Let $X$ be a Fano manifold with nef tangent bundle satisfying the hypothesis of the Main Theorem. Let $V$ be a rank-2 holomorphic vector bundle on $\mathcal{K}$ whose projectivization is isomorphic to the holomorphic $\mathbb{P}^{1}$-bundle $\rho: \mathcal{U} \longrightarrow$ $\mathcal{K}$. Then, $V$ is stable.

Remarks. (1) Since $b_{2}(\mathcal{K})=1$ by Lemma $(2.1 .1), \mathcal{K}$ is of Picard number 1 , and stability of a vector bundle is equivalently defined in terms of any positive holomorphic line bundle on $\mathcal{K}$.

(2) $V$ is uniquely defined up to a tensor product with some holomorphic line bundle $L$ on $\mathcal{K}$. For any choices of $V$ and $L$, the stability of $V$ is equivalent to the stability of $V \otimes L$.

Proof of Proposition (2.1.1). Denote by $\mathcal{V}$ the sheaf of germs of holomorphic sections of $V$. Suppose $V$ is not stable over $\mathcal{K}$. Then, there exists a maximal destabilizing coherent subsheaf $\mathcal{F} \subset \mathcal{V}$ of rank 1 . We are going to derive a contradiction at a generic point $[C] \in \mathcal{K}$ from the existence of $\mathcal{F}$.

Now choose $[C] \in \mathcal{K}$ such that the rank-1 coherent subsheaf $\mathcal{F} \subset \mathcal{V}$ is locally free at $[C]$. By maximality of $\mathcal{F}$, the latter corresponds to a holomorphic line subbundle of $V$ in a neighborhood of $[C]$. Choose any point $x \in C$ and consider the rational curve on $\mathcal{K}$ given by $\mathcal{K}_{x}:=\rho\left(\mathcal{U}_{x}\right) \subset \mathcal{K}$. Consider the restriction of the holomorphic $\mathbb{P}^{1}$-bundle $\rho: \mathcal{U} \longrightarrow \mathcal{K}$ to $\mathcal{K}_{x}$. Let $\nu: \mathcal{K}_{x}^{\prime} \longrightarrow \mathcal{K}_{x} \subset \mathcal{K}$ be the normalization of $\mathcal{K}_{x}$. We have $\mathcal{K}_{x} \cong \mathbb{P}^{1}$ by Lemma (1.2.2). Pulling back $\rho: \mathcal{U} \longrightarrow \mathcal{K}$ by $\nu$, we obtain a $\mathbb{P}^{1}$-bundle $\nu^{*} \mathcal{U}$ which is the projectivization $\mathbb{P}\left(\nu^{*} V\right)$. Then, $\nu^{*} \mathcal{U}_{x}$ defines a holomorphic section of $\nu^{*} \mathcal{U}$ over $\mathcal{K}_{x}^{\prime}$. Over $\mathcal{K}_{x} \cong \mathbb{P}^{1}, \nu^{*} V$ splits into the direct $\operatorname{sum} \mathcal{O}(a) \oplus \mathcal{O}(b)$ for some integers $a \geq b$. We proceed to show that $a>b$.

Pulling back $\mu: \mathcal{U} \longrightarrow X$ by $\nu$, we obtain a holomorphic map $\nu^{*} \mu: \nu^{*} \mathcal{U} \longrightarrow X$ which is generically of rank 2 and which collapses $\nu^{*} \mathcal{U}_{x}$ into a point $x$. It follows that the normal bundle of $\nu^{*} \mathcal{U}_{x}$ in $\nu^{*} \mathcal{U}$ is negative. From this it follows that $a>b$, as desired. By Lemma (2.1.1), $\mathbb{P}(\mathcal{O}(a) \oplus \mathcal{O}(b)), a>b$, has a unique section $\mathbb{P}(\mathcal{O}(a))$ which is exceptional, $\mathbb{P}(\mathcal{O}(a))=\nu^{*} \mathcal{U}_{x}$.

Now $\nu^{*} V$ over $\mathcal{K}_{x} \cong \mathbb{P}^{1}$ contains a holomorphic line subbundle $L$ whose associated coherent subsheaf $\mathcal{L}$ agrees with $\nu^{*} \mathcal{F}$ at a point $z$ of $\mathcal{K}_{x}^{\prime}$ where $\mathcal{F}$ is locally free at $\nu(z)$. Let $\omega$ be a $d$-closed positive $(1,1)$-form on $\mathcal{K}$. Since $\mathcal{F}$ is a destabilizing 
coherent subsheaf, we must have

$$
c_{1}(\mathcal{F}) \wedge \omega^{n-1} \geq \frac{1}{2} c_{1}(\mathcal{V}) \wedge \omega^{n-1} .
$$

Since $b_{2}(\mathcal{K})=1$, the $(n-1, n-1)$-closed positive current defined by the cycle $\left[\mathcal{K}_{x}\right]$ must be cohomologous to $a \cdot \omega^{n-1}$ for some constant $a>0$. We have always

$$
c_{1}(L) \geq c_{1}\left(\nu^{*} \mathcal{F}\right)=c_{1}(\mathcal{F}) \wedge a \cdot \omega^{n-1} .
$$

(For the definition of the inverse image sheaf $\nu^{*} \mathcal{F}$, cf. [GR $\S 2.6$, p.18]. In $(*)$ the last equality can be seen using a resolution of $\mathcal{F}$ on $\mathcal{K}$ by locally free sheaves.) From the Grothendieck splitting $\nu^{*} V \cong \mathcal{O}(a) \oplus \mathcal{O}(b), a>b$, for any integer $c \geq$ $\frac{1}{2}(a+b)$, any non-trivial homomorphism $\sigma \in \Gamma\left(\mathcal{K}_{x}^{\prime}, \operatorname{Hom}\left(\mathcal{O}(c), \nu^{*} V\right)\right)$ must map $\mathcal{O}(c)$ into $\mathcal{O}(a)$. It follows that at $[C] \in \mathcal{K}$, where $\mathcal{F}$ corresponds to a holomorphic line subbundle $F$ in a neighborhood of $[C]$, the fiber $F_{[C]}$ must correpond to the component of maximal degree under restriction to $\mathcal{K}_{x}$ and pulling back by the normalization $\nu$. Now at $[C] \in \mathcal{K}_{x}$, the component of maximal degree correponds precisely to the point $w \in \rho^{-1}([C]) \cap \mathcal{U}_{x}$. Varying the base point $x \in C$, we obtain a one-parameter family of of rational curves $\mathcal{K}_{y}$ passing through $[C]$. The corresponding points $w(y)$ vary, contradicting the fact that $F_{[C]}$ is well-defined. As a consequence, the destabilizing coherent subsheaf $\mathcal{F} \subset \mathcal{V}$ cannot possibly exist. In other words, we have proven that $V$ is stable over $\mathcal{K}$, as desired.

(2.2). A generic $\mathcal{K}$-rational curve on $X$ is standard. Thus, the analytic subvariety $S \subset \mathcal{U}$ corresponding to points where $d \mu$ is not submersive is a subvariety of codimension $\geq 2$. It follows that for a generic point $x \in X$ any local irreducible branch of any $\mathcal{K}$-rational curve passing through $x$ is smooth at $x$. We call such a point $x$ a $\mathcal{K}$-generic point. We proceed now to establish an a priori bound on degrees of VMRTs at $\mathcal{K}$-generic points, as follows.

Proposition (2.2.1). Let $X$ be a Fano manifold with nef tangent bundle such that $b_{2}(X)=b_{4}(X)=1$. Suppose $X$ admits a minimal rational component $\mathcal{K}$ whose generic varieties of minimal rational curves are 1-dimensional. Let $C \subset X$ be a standard minimal rational curve on $X$ such that a generic point $x$ of $C$ is $\mathcal{K}$ generic. Then, the variety of minimal rational tangents $\mathcal{C}_{x} \subset \mathbb{P} T_{x}(X)$ is a rational curve of degree $d<4$.

Proof. Recall that $V$ is a rank-2 holomorphic vector bundle such that $\mathbb{P} V$ is isomorphic to the universal family $\rho: \mathcal{U} \longrightarrow \mathcal{K}$. Let $\omega$ be any Kähler form on $X$. By Proposition (2.1.1), $V$ is stable. By Uhlenbeck-Yau [UY] there exists on $V$ a Hermitian-Einstein metric with respect to $\omega$. It follows that (cf. Siu [Si (1.8), pp.23-24])

$$
c_{1}^{2}(V) \wedge[\omega]^{n-2} \leq 4 c_{2}(V) \wedge[\omega]^{n-2},
$$

where $[\omega]$ denotes the cohomology class in $H^{2}(\mathcal{K}, \mathbb{R})$ corresponding to $\omega$. To prove Proposition (2.2.1) we are going to show first of all that the Chern class inequality is violated whenever $d>4$.

Denote by $\epsilon$ a nonzero element of $H^{2}(X, \mathbb{Q})$ such that, with respect to the canonical pairing between $H^{2}(X, \mathbb{Q})$ and $H_{2}(X, \mathbb{Q})$, we have $\epsilon([C])=1$ for the second homology class $[C]$ defined by any minimal rational curve $C$. Similarly let $\delta$ be a nonzero element of $H^{2}(X, \mathbb{Q})$ such that, for the second homology class $\left[\mathcal{K}_{x}\right]$ defined by the rational curve $\mathcal{K}_{x}=\rho\left(\mathcal{U}_{x}\right)$ for a $\mathcal{K}$-generic point $x$ on $X$, we have $\delta\left(\left[\mathcal{K}_{x}\right]\right)=1$. 
$H^{2}(\mathcal{U}, \mathbb{Q})$ is 2 -dimensional, generated by $\mu^{*} \epsilon$ and $\rho^{*} \delta$. To avoid clumsy notations we will sometimes use $\epsilon$ resp. $\delta$ to denote $\mu^{*} \epsilon$ resp. $\rho^{*} \delta$, etc. $H^{4}(\mathcal{U}, \mathbb{Q})$ is also 2-dimensional, generated by $\epsilon^{2}, \epsilon \delta$ and $\delta^{2}$, where $\epsilon^{2}$ stands for $\left(\mu^{*} \epsilon\right)^{2}$, etc. There is therefore a non-trivial linear relation among $\epsilon^{2}, \epsilon \delta$ and $\delta^{2}$. This linear relation will play an important role in the proof of Proposition (2.2.1).

We will break down the proof of $d<4$ into a number of steps. The point is to exploit the Chern class inequality arising from the stability of $V$. The Chern class $c_{1}^{2}(V)-4 c_{2}(V)$ on $\mathcal{K}$ can be computed when we lift it to $\mathcal{U}$, in terms of generators $\epsilon^{2}, \epsilon \delta$ and $\delta^{2}$ of $H^{4}(\mathcal{U}, \mathbb{Q}) \cong \mathbb{Q}^{2}$. The expression is useful only if we know a nontrivial linear relation on the generators, which is an intermediate objective in the ensuing computation. We have

Lemma (2.2.1). Let $x \in X$ be a $\mathcal{K}$-generic point. Suppose for the normalization $\nu: \mathbb{P}^{1} \longrightarrow \mathcal{K}_{x}$ we have $\nu^{*} V \cong \mathcal{O}(a) \oplus \mathcal{O}(b), a>b$. Then, the total Chern class $c\left(\rho^{*} V\right)$ is given by

$$
c\left(\rho^{*} V\right)=1+(a+b) \delta+\left((a-b) \epsilon \delta-\epsilon^{2}+(a b) \delta^{2}\right) .
$$

Proof. Denote by $E \subset \rho^{*} V$ the tautological line subbundle and by $P$ the quotient $\rho^{*} V / E$. We have $c\left(\rho^{*} V\right)=c(E) \cdot c(P)$. Fix a standard rational curve $C$ which contains a $\mathcal{K}$-generic point $x$. We consider Chern classes of restrictions of $E$ and $Q$ to the tautological lifting $\hat{C} \subset \mathcal{U}$ and to $\mathcal{U}_{x} \subset \mathcal{U}$. Both $\hat{C}$ and $\mathcal{U}_{x}$ are smooth rational curves on $\mathcal{U}$. We will denote by $\mathcal{O}(s)$ the holomorphic line bundle on either smooth rational curve of degree $s$. Since $\left.E\right|_{\hat{C}}$ is the tautological line bundle on $\hat{C}=\mathbb{P} V_{[C]}$, we have $\left.E\right|_{\hat{C}} \cong \mathcal{O}(-1)$. Recall that for a normalization $\nu: \mathbb{P}^{1} \longrightarrow \mathcal{K}_{x}, \nu^{*} \mathcal{U}_{x}$ is an exceptional curve on $\nu^{*} \mathcal{U}$ which is at the same time a holomorphic section of $\nu^{*} \mathcal{U}$ over $\mathbb{P}^{1}$, so that by Lemma $(2.1 .1), \mathcal{U}_{x}$ corresponds to $\mathbb{P}(\mathcal{O}(a))$. In other words, $\left.E\right|_{\mathcal{U}_{x}} \cong \mathcal{O}(a)$. For the quotient bundle $P$ we obviously have $\left.P\right|_{\hat{C}}=\rho^{*} V_{[C]} /\left.E\right|_{\hat{C}} \cong$ $\mathcal{O}^{2} / \mathcal{O}(-1) \cong \mathcal{O}(1)$. On the other hand, $\left.Q\right|_{\mathcal{U}_{x}}=\left.\left(\rho^{*} V / E\right)\right|_{\mathcal{U}_{x}} \cong(\mathcal{O}(a) \oplus \mathcal{O}(b)) / \mathcal{O}(a)$ $\cong \mathcal{O}(b)$. It follows that

$$
\begin{aligned}
& c(E)=1+a \delta-\epsilon, \\
& c(P)=1+b \delta+\epsilon,
\end{aligned}
$$

and so

$$
\begin{aligned}
c\left(\rho^{*} V\right) & =c(E) \cdot c(P)=(1+a \delta-\epsilon)(1+b \delta+\epsilon) \\
& =1+(a+b) \delta+\left((a-b) \epsilon \delta-\epsilon^{2}+(a b) \delta^{2}\right),
\end{aligned}
$$

as desired.

Next, we consider the holomorphic $\mathbb{P}^{1}$-bundle $\mu: \mathcal{U} \rightarrow X$. While we do not know that it satisfies the hypothesis of Lemma (1.3.1), we can still consider a rank2 holomorphic bundle when we restrict $\mu: \mathcal{U} \rightarrow X$ over a minimal rational curve. Computation of Chern classes, together with Lemma (2.2.1), will lead to the desired linear relation to be given in Lemma (2.2.3). Let $C \subset X$ be a standard minimal rational curve belonging to $\mathcal{K}$, and let $\sigma: \mathbb{P}^{1} \rightarrow C$ be a normalization. Recall that $\mu: \mathcal{U} \rightarrow X$ is a holomorphic $\mathbb{P}^{1}$-bundle. Thus, $\sigma^{*} \mathcal{U}$ is a holomorphic $\mathbb{P}^{1}$-bundle.

Now let $\sigma: \mathbb{P}^{1} \rightarrow C$ be a normalization. ( $C$ may carry nodal singularities.) $\sigma^{*} \mathcal{U}=\mathbb{P}\left(\sigma^{*} W\right)$ is a holomorphic $\mathbb{P}^{1}$-bundle with a holomorphic section $\sigma^{*} \hat{C}$ over 
$\mathbb{P}^{1}$ which is at the same time an exceptional curve. It follows that

$$
\sigma^{*} \mathcal{U} \cong \mathbb{P}(\mathcal{O}(e+c) \oplus \mathcal{O}(c))
$$

where $c$ is some integer and $-e$ is the degree of the normal bundle of $\sigma^{*} \hat{C}$ in the surface $\sigma^{*} \mathcal{U}$. From the universal family $\rho: \mathcal{U} \rightarrow \mathcal{K}$ we know that the normal bundle $N$ of $\hat{C}$ in $\mathcal{U}$ is holomorphically trivial. The surface $\mu^{-1}(C)$ is smooth in a neighborhood of the tautological lifting $\hat{C}$ of $C$. Let $\Omega$ be a smooth neighborhood of $\hat{C}$ in $\mu^{-1}(C)$. The restriction $R$ of the normal bundle of $\Omega$ in $\mathcal{U}$ is isomorphic to the pull-back of $\sigma^{*} T_{X} / T_{\mathbb{P}^{1}}$ under the biholomorphic map $\lambda: \hat{C} \cong \mathbb{P}^{1}$ such that $\sigma \circ \lambda=\left.\mu\right|_{\hat{C}}$. It follows that $R \cong \mathcal{O}(1) \oplus \mathcal{O}^{n-2}$ and that

$$
N \cong \mathcal{O}^{n-1} / R \cong \mathcal{O}^{n-1} /\left(\mathcal{O}(1) \oplus \mathcal{O}^{n-2}\right) \cong \mathcal{O}(-1),
$$

so that $e=1$. Over $\mathbb{P}^{1}$ the holomorphic $\mathbb{P}^{1}$-bundle $\sigma^{*} \mathcal{U}$ is isomorphic to the projectivization $\mathbb{P} W$ of a rank-2 holomorphic vector bundle $W$, where $W \cong$ $\mathcal{O}(c+1) \oplus \mathcal{O}(c)$ for some integer $c$. Let $F \subset W$ be the tautological line bundle and $Q=W / F$ the quotient bundle. Obviously $\left.F\right|_{\left(\sigma^{*} \mathcal{U}\right)_{z}} \cong \mathcal{O}(-1)$ and $\left.Q\right|_{\left(\sigma^{*} \mathcal{U}\right)_{z}} \cong \mathcal{O}(1)$ for any $z \in \mathbb{P}^{1}$. From $W \cong \mathcal{O}(c+1) \oplus \mathcal{O}(1)$ and $\hat{C}=\mathbb{P}(\mathcal{O}(c+1))$ it follows that $\left.F\right|_{\hat{C}} \cong \mathcal{O}(c+1)$ and $\left.Q\right|_{\hat{C}} \cong \mathcal{O}(c)$.

We are now ready to compute $c\left(\nu^{*} W\right)$, to deduce

Lemma (2.2.2). Let $\zeta: \sigma^{*} \mathcal{U} \rightarrow \mathcal{U}$ be the canonical map. Then, $\zeta^{*}\left(\epsilon \delta-\delta^{2}\right)=0$ in $H^{4}\left(\sigma^{*} \mathcal{U}, \mathbb{Q}\right) \cong \mathbb{Q}^{2}$.

Proof. From preceding computations we have

$$
\begin{gathered}
c(F)=1-\zeta^{*} \delta+(c+1) \zeta^{*} \epsilon, \\
c(Q)=1+\zeta^{*} \delta+c \zeta^{*} \epsilon,
\end{gathered}
$$

so that

$$
\begin{aligned}
& c\left(\nu^{*} W\right)=c(F) \cdot c(Q)=\left(1-\zeta^{*} \delta+(c+1) \zeta^{*} \epsilon\right)\left(1+\zeta^{*} \delta+c \zeta^{*} \epsilon\right) \\
& \quad=1+(2 c+1) \zeta^{*} \epsilon+\zeta^{*}\left(\epsilon \delta-\delta^{2}+c(c+1) \epsilon^{2}\right) .
\end{aligned}
$$

Since $\mu \zeta\left(\sigma^{*} \mathcal{U}\right)=C$ is a curve, we have $\zeta^{*}\left(\epsilon^{2}\right)=0$. On the other hand, since $W$ is a holomorphic vector bundle over $\mathbb{P}^{1}$, we have $c_{2}\left(\nu^{*} W\right)=\nu^{*} c_{2}(W)=0$, so that

$$
0=c_{2}\left(\nu^{*} W\right)=\zeta^{*}\left(\epsilon \delta-\delta^{2}\right)
$$

as desired.

From Lemmas (2.2.1) and (2.2.2) we deduce a nontrivial linear relation among the 3 generators of $H^{4}(\mathcal{U}, \mathbb{Q})$, as follows.

Lemma (2.2.3). In the notations of Lemma (2.2.1) the difference $a-b$ agrees with the degree $d$ of $\mathcal{C}_{x}$ in $\mathbb{P} T_{x}(X)$ for any $\mathcal{K}$-generic point $x \in X$. Furthermore, we have

$$
\epsilon^{2}-d \epsilon \delta+d \delta^{2}=0
$$

as cohomology classes in $H^{4}(\mathcal{U}, \mathbb{Q}) \cong \mathbb{Q}^{2}$.

Proof. Since $H^{4}(\mathcal{U}, \mathbb{Q}) \cong \mathbb{C}^{2}$, the three generators $\epsilon^{2}, \epsilon \delta$ and $\delta^{2}$ must satisfy a linear relation, unique up to a scalar multiple. Denote for the time being by $h$ the difference $a-b$, in the notations of Lemma (2.2.1). Observe that $c_{2}\left(\rho^{*} V\right) \in$ $\rho^{*} H^{4}(\mathcal{K}, \mathbb{Q})$, and the latter is 1-dimensional, generated by $\delta^{2}$. From the formula in Lemma (2.2.1) for the total Chern class of $\rho^{*} V$ the linear relation can be written 
as $\epsilon^{2}-h \epsilon \delta+g \delta^{2}=0$ for some rational number $g$. We claim that $g=h$. Pulling back to $\sigma^{*} \mathcal{U}$ by $\zeta$, we have

$$
0=\zeta^{*}\left(\epsilon^{2}-h \epsilon \delta+g \delta^{2}\right)=-h \zeta^{*}(\epsilon \delta)+g \zeta^{*}\left(\delta^{2}\right) .
$$

On the other hand, by $(2.2 .2) \zeta^{*}(\epsilon \delta)=\zeta^{*}\left(\delta^{2}\right)$, so that

$$
0=(g-h) \zeta^{*}\left(\delta^{2}\right) \text {. }
$$

Since $\delta^{2}$ is represented by some closed positive $(2,2)$ form, and $\rho \zeta\left(\sigma^{*} \mathcal{U}\right)$ is a surface in $\mathcal{K}$, we have $\zeta^{*}\left(\delta^{2}\right) \neq 0$, from which it follows that $g=h$, as claimed.

For the proof of Lemma (2.2.3) it remains to show that $h$ agrees with the degree $d$ of $\mathcal{C}_{x} \subset \mathbb{P} T_{x}(X)$ for a $\mathcal{K}$-generic point $x \in X$. Recall that $\nu: \mathbb{P}^{1} \longrightarrow \mathcal{K}_{x}$ denotes a normalization of the rational curve $\mathcal{K}_{x} \subset \mathcal{K}$. The tangent map $\tau_{x}: \mathcal{U}_{x}\left(\cong \mathbb{P}^{1}\right) \longrightarrow \mathcal{C}_{x}$ is a normalization. $\mathcal{U}_{x}$ parametrizes a $\mathbb{P}^{1}$-family of germs of smooth curves $D_{x}(z)$ at $x \in X$ such that at $z \in \mathcal{U}_{x}, \tau_{x}(z)$ is tangent to $D_{x}(z)$. It follows that the normal bundle $N$ of $\nu^{*} \mathcal{U}_{x}$ in $\nu^{*} \mathcal{U}$ agrees with the pull-back under $\tau_{x}$ of the tautological line bundle on $\mathbb{P} T_{x}(X)$. Thus, the degree of $N$ agrees with $-d$. On the other hand, from $\nu^{*} \mathcal{U}=\nu^{*} \mathbb{P}(V) \cong \mathbb{P}(\mathcal{O}(a) \oplus \mathcal{O}(b))$ we know that $h=b-a$. In other words, $h=d$, as desired.

Proof of Proposition (2.2.1), continued. Since $V$ is a stable rank-2 holomorphic vector bundle on $\mathcal{K}$, we must have

$$
c_{1}^{2}(V) \leq 4 c_{2}(V)
$$

as cohomology classes in the 1 -dimensional $\mathbb{Q}$-vector space $H^{4}(\mathcal{K}, \mathbb{Q})$, where $\delta^{2}$ is taken to be positive (cf. Siu [Si, (1.8), pp.23-24]). Thus,

$$
\begin{aligned}
0 & \leq 4 c_{2}(V)-c_{1}^{2}(V)=4\left(a b \delta^{2}-\epsilon^{2}+d \epsilon \delta\right)-(a+b)^{2} \delta^{2} \\
& =\left(4 a b-(a+b)^{2}\right) \delta^{2}-4\left(\epsilon^{2}-d \epsilon \delta+d \delta^{2}\right)+4 d \delta^{2} .
\end{aligned}
$$

By Lemma (2.2.3) the second last term vanishes, and we conclude

$$
0 \leq\left(2 a b-a^{2}-b^{2}\right) \delta^{2}+4 d \delta^{2}=\left(4 d-(a-b)^{2}\right) \delta^{2}=\left(4 d-d^{2}\right) \delta^{2} .
$$

In other words, $d^{2} \leq 4 d$, i.e., $d \leq 4$. If we have the strict inequality $c_{1}^{2}(V)<4 c_{2}(V)$, then $d^{2}<4 d$, i.e. $d<4$. On the other hand, from the proof of the inequality $c_{1}^{2}(V) \leq 4 c_{2}(V)$ using a Hermitian-Einstein metric $h$ on $V, c_{1}^{2}(V)=4 c_{2}(V)$ if and only if $c_{1}^{2}(V, h)=4 c_{2}(V, h)$ as $(2,2)$-forms (cf. Siu [Si, loc. cit.]). More precisely, let $\omega$ be a Kähler form on $X$, and let $(V, h)$ be Hermitian-Einstein with respect to $\omega$ with Einstein constant $\lambda$. Let $x \in X$, let $\left(z_{i}\right), 1 \leq i \leq m$, be a system of holomorphic local coordinates at $x$ such that $\frac{\partial}{\partial z_{i}}$ is orthonormal at $x$, and let $\left(e_{\alpha}\right)$ be on orthonormal basis of $V_{x}$. Then, the coefficient $A$ of $\frac{1}{4 \pi^{2}} \prod_{\alpha=1}^{m}\left(i d z^{\alpha} \wedge d \bar{z}^{\alpha}\right)$ in $\left[\left(4 c_{2}(V, h)-c_{1}^{2}(V, h)\right) \wedge \omega^{m-2}\right](x)$ is given by

$$
A=\frac{1}{2} \sum_{\alpha, \beta, i, j}\left|F_{\alpha \bar{\alpha} i \bar{j}}-F_{\beta \bar{\beta} i \bar{j}}\right|^{2}+2 \sum_{\alpha \neq \beta, i, j}\left|F_{\alpha \bar{\beta} i \bar{j}}\right|^{2} \geq 0 .
$$

Since $(\operatorname{det}(V), \operatorname{det}(h))$ is also Hermitian-Einstein, $A=0$ if and only if

$$
F_{\alpha \bar{\beta} i \bar{j}}=\frac{\lambda}{m} \delta_{\alpha}^{\beta} \delta_{i}^{j}
$$

Thus, $c_{1}^{2}(V)=4 c_{2}(V)$ implies that locally $(V, h) \otimes\left(\operatorname{det}(V)^{-\frac{1}{2}}, \operatorname{det}(h)^{-\frac{1}{2}}\right)$ is flat, $\left(\operatorname{det}(V)^{-\frac{1}{2}}, \operatorname{det}(h)^{-\frac{1}{2}}\right)$ being a local square root of the Hermitian holomorphic line bundle $\left(\operatorname{det}\left(V^{*}\right), \operatorname{det}(h)^{-1}\right)$. In other words, $(V, h)$ is locally isomorphic to the tensor 
product of a Hermitian holomorphic line bundle and the flat rank-2 holmorphic vector bundle. Thus, $\mathbb{P} V$ is a locally constant projective unitary bundle. Since $X$ is simply-connected, $\mathbb{P} V$ must be trivial. $V$ must therefore be isomorphic on $\mathcal{K}$ to the tensor product $E \otimes \mathcal{O}^{2}$ for some holomorphic line bundle $E$ on $X$, and as such cannot be stable. We have therefore proven that the stable vector bundle $V$ on $\mathcal{K}$ actually satisfies $c_{1}^{2}(V)<4 c_{2}(V)$, and that $d<4$.

\section{RECOVERING the RATional homogeneous StRuCture FROM 1-DIMENSIONAL VMRTS}

(3.1). Let $X$ be a Fano manifold with nef tangent bundle satisfying the hypothesis of the Main Theorem. By Proposition (3.1.2), the VMRT $\mathcal{C}_{x} \subset \mathbb{P} T_{x}(X)$ at a generic point $x \in X$ is a rational curve of degree 1,2 or 3 . To complete the proof of the Main Theorem, we will show that they correspond precisely to the cases (a), (b) and (c) in the statement there.

We will need two types of preliminary results. On the one hand, we will need results regarding distributions spanned by, or more generally containing, VMRTs, concerning their integrability or non-integrability. They are essentially contained in HM2 and stated more precisely in HM4. On the other hand, we will need to recover the structure of irreducible Hermitian symmetric spaces of rank $\geq 2$ and homogeneous contact structures on Fano manifolds of Picard number 1 carrying special types of VMRTs. The former is contained in Hwang-Mok [HM1], and the latter in Hong [Ho]. Regarding distributions arising from VMRTs we have

Proposition (3.1.1) (Hwang-Mok [HM2, [HM3])). Let X be a Fano manifold of Picard number 1 , and $\mathcal{K}$ be a minimal rational component on $X$. Suppose a generic VMRT $\mathcal{C}_{x}$ associated to $\mathcal{K}$ is linearly degenerate. Let $W$ be a proper meromorphic distribution on $X$ which contains the linear span of $\mathcal{C}_{x}$ at a generic point. Then, $W$ cannot be integrable.

Proposition (3.1.2) (Hwang-Mok [HM2], [HM3])). Let $X$ be a uniruled projective manifold and $\mathcal{K}$ be a minimal rational component on $X$. Suppose a generic $V M R T \mathcal{C}_{x}$ associated to $\mathcal{K}$ is linearly degenerate. Let $D$ be the proper mermorphic distribution on $X$ which is linearly spanned by $\mathcal{C}_{x}$ at a generic point $x \in X$. Denote by $\mathcal{T}_{x} \subset \mathbb{P}\left(\Lambda^{2} D_{x}\right)$ the tangent variety of $\mathcal{C}_{x}$. Suppose $\mathcal{T}_{x}$ is linearly non-degenerate in $\mathbb{P}\left(\Lambda^{2} D_{x}\right)$ at a generic point. Then, $D$ is integrable. The former is always the case whenever $\mathcal{C}_{x}$ is irreducible and the projective second fundamental form of $\mathcal{C}_{x} \subset \mathbb{P} D$ at a generic smooth point $[\alpha]$ of $\mathcal{C}$ is surjective. In particular, $D$ is always integrable whenever $\mathcal{C}_{x} \subset \mathbb{P} D$ is a linearly non-degenerate irreducible hypersurface.

Regarding the recovery of geometric structures using VMRTs in the case of Hermitian symmetric spaces and Fano homogeneous contact manifolds, we have, first of all,

Proposition (3.1.3) (from Hwang-Mok [HM1]). Let $S$ be an irreducible Hermitian symmetric space of compact type and of rank $\geq 2$, and let $\mathcal{C}_{o} \subset \mathbb{P} T_{o}(S)$ be the $V M R T$ of $S$ at a reference point $o \in S$. Let $X$ be a Fano manifold of Picard number 1 whose VMRT $\mathcal{C}_{x} \subset \mathbb{P} T_{x}(S)$ at $x \in X$ is isomorphic to $\mathcal{C}_{o} \subset \mathbb{P} T_{o}(S)$ as a projective subvariety for $x$ lying outside a subvariety $Z \subset X$ of codimension $\geq 2$. Then, $X$ is biholomorphically isomorphic to $S$.

In HM1 the result is formulated in terms of $S$-structures, and more generally for $G$-structures corresponding to irreducible representations of reductive Lie groups $G$ 
on tangent spaces. The fact that VMRTs on $X$ agree with varieties of highest weight tangents of $G$ was part of the result there. The exceptional set $Z$ in Proposition (3.1.3) can be removed by a Hartogs-type extension theorem of $G$-structures, cf. Hwang-Mok HM2].

Finally, we will need the following slight modification of a result of Hong's ( $[\mathrm{Ho}]$ which recovers the structure of a Fano homogeneous contact manifold of Picard number 1 from its VMRTs. (As in [HM1], the original hypothesis was one on $G$ structures. The fact that the VMRTs agree with varieties of highest weight tangents of associated reductive representations was part of the theorem.)

Proposition (3.1.4) (from Hong $[\mathrm{Ho}]$ ). Let $S$ be a Fano homogeneous contact manifold of Picard number 1 different from an odd-dimensional projective space. Let $\mathcal{C}_{o} \subset \mathbb{P} T_{o}(S)$ be the VMRT of $S$ at a reference point $o \in S$. Let $X$ be a Fano manifold of Picard number 1 whose $\operatorname{VMRT} \mathcal{C}_{x} \subset \mathbb{P} T_{x}(S)$ at $x \in X$ is isomorphic to $\mathcal{C}_{o} \subset \mathbb{P} T_{o}(S)$ as a projective subvariety for $x$ lying outside a subvariety $Z \subset X$ of codimension $\geq 2$. Denoting by $D$ the meromorphic distribution on $X$ spanned by the VMRTs, assume that the Frobenius form $\varphi: \Lambda^{2} D \longrightarrow T_{X} / D$ is everywhere non-degenerate on $X-Z$. Suppose furthermore that at every point $x \in X-Z$, a generic minimal rational curve passing through $x$ lies on $X-Z$. Then, $X$ is biholomorphically isomorphic to $S$.

From the hypothesis of Proposition (3.1.4) one can construct Cartan connections on $X-Z$ adapted to the contact structures, and apply the vanishing-type results of Hong $[\mathrm{Ho}$, on (higher-order) curvature tensors. We note that at $x \in X-Z$ the vanishing-type results already apply when a generic minimal rational curve passing through $x$ lies on $X-Z$, as is our hypothesis. Unlike the case of $S$-structures arising from irreducible Hermitian symmetric spaces of rank $\geq 2$, there is no Hartogs-type extension theorem across $Z$. Nonetheless, $X-Z$ is simply-connected, and the local identification of $X-Z$ with the model space $S$ as given by Hong [Ho leads readily to a birational map between $X$ and $S$, which must then be a biholomorphic map by Hwang-Mok HM4, Proposition (5.4)].

(3.2). We proceed with the reconstruction of the 3 rational homogeneous manifolds listed in Theorem 1 from VMRTs.

Proof of the Main Theorem. Let $X$ be a Fano manifold with nef tangent bundle such that $b_{2}(X)=b_{4}(X)=1$ and such that $X$ admits a minimal rational component whose associated VMRTs are generically 1-dimensional. By Proposition (3.1.2) we know that the generic $\operatorname{VMRT} \mathcal{C}_{x} \subset \mathbb{P} T_{x}(X)$ is of degree $d=1,2,3$.

Case 1: $d=1$. At a generic $x \in X, \mathcal{C}_{x} \subset \mathbb{P} T_{x}(X)$ is a projective line. The meromorphic distribution $D$ on $X$ spanned at generic points by $\mathcal{C}_{x}$ must then be integrable by Proposition (3.1.2). Since $X$ is of Picard number 1, by Proposition (3.1.1) $D$ cannot be integrable, unless $D=T_{X}$. Thus, $X$ is 2 -dimensional. As the tangent bundle of $X$ is nef, and $X$ is of Picard number 1 , it must be the projective plane $\mathbb{P}^{2}$.

In what follows $D$ will always stand for the meromorphic distribution on $X$ spanned at generic points by VMRTs.

Case 2: $d=2$. Since $\mathbb{P}^{2}$ is the only projective surface with nef tangent bundle and of Picard number 1 , in which case $d=1, X$ must be of dimension $\geq 3$ whenever $d>1$. Let $L$ be the tautological line bundle on $\mathbb{P} T_{X}$, and let $x$ be a generic point on $X$. Since the subset of $\mathcal{U}$ corresponding to singular points of minimal rational curves is 
of codimension $\geq 2$ in $\mathcal{U}$ and $\mathcal{U}_{x}$ is 1-dimensional, for $x \in X$ generic the tangent map $\tau_{x}: \mathcal{U}_{x} \rightarrow \mathbb{P} T_{x}(X)$ is a holomorphic map. We have $\mathcal{U}_{x} \cong \mathbb{P}^{1}$ and $\tau_{x}^{*}\left(L^{*}\right) \cong \mathcal{O}(d)$. Since $H^{o}\left(\mathbb{P}^{1}, \mathcal{O}(d)\right) \cong \mathbb{C}^{d+1}$, for $d=2$ the linear span $D_{x} \subset T_{x}(X)$ must be either 2 or 3 -dimensional. If $D$ is of rank 2 , then $\mathcal{C}_{x}=\mathbb{P} D_{x}$ is a projective line with multiplicity 2 , and $D$ must again be integrable, leading to a contradiction. If $D$ is of rank 3 , then $\mathcal{C}_{x} \subset \mathbb{P} D_{x} \cong \mathbb{P}^{2}$ is an irreducible curve (hypersurface) of degree 2 , and $D$ must be integrable, by Proposition (3.1.2). It follows again by Proposition (3.1.1) that $X$ is of dimension 3. From the classification of 3-dimensional Fano manifolds with nef tangent bundle it follows that $X$ is biholomorphic to the 3 dimensional hyperquadric $Q^{3}$.

We include here another proof that $X \cong Q^{3}$, involving an argument using geometric structures, since a variation of this argument will be needed in the case $d=3$. We have reduced to the case where $X$ is 3 -dimensional and the generic VMRT is a non-degenerate quadric in $\mathbb{P} T_{x}(X) \cong \mathbb{P}^{2}$. By Proposition (3.1.3) it suffices to show that this is the case for every $x \in X-Z$ for some subvariety $Z \subset X$ of codimension $\geq 2$. Let $E \subset X$ be the smallest subvariety such that the tangent map $\tau: \mathcal{U} \rightarrow \mathbb{P} T_{X}$ is holomorphic over $X-E$ and such that $\tau_{x}: \mathcal{U}_{x} \rightarrow \mathbb{P} T_{x}(X)$ is a holomorphic embedding of $\mathcal{U}_{x} \cong \mathbb{P}^{1}$ onto a non-degenerate quadric curve in $\mathbb{P} T_{x}(X) \cong \mathbb{P}^{2}$ for $x \in X-E$. We argue that $E$ must be of codimension $\geq 2$ in $X$. Suppose otherwise. Then, $E$ contains an irreducible hypersurface $H$ of $X$. Since $X$ is of Picard number 1, any minimal rational curve $C$ must intersect $H$. It follows that at a generic point $y$ of $H$, there exists a standard minimal rational curve $C$ such that $C$ intersects $H$ at $y$. We argue that $\mathcal{C}_{y} \subset \mathbb{P} T_{y}(X)$ must then be a nondegenerate quadric curve, and that the tangent map $\tau$ must be an embedding in a neighborhood of $\mathcal{U}_{y} \subset \mathcal{U}$. Recall that $\hat{C} \subset \mathcal{U}$ denotes the canonical lifting of $C$ to the universal family. We denote by $C^{\prime} \subset \mathbb{P} T_{X}$ the tautological lifting of $C$. The tangent map $\tau$ is a holomorphic immersion in a neighborhood of $\hat{C}$, mapping the latter onto $C^{\prime} . C^{\prime} \subset \mathbb{P} T_{X}$ is always immersed. To avoid clumsy notations we assume that it is embedded. The general case will follow with obvious modifications, which we omit. Under the assumption that $C^{\prime}$ is embedded, $\tau$ is a holomorphic embedding in a neighborhood $\mathcal{D}$ of $\hat{C}$ in $\mathcal{U} . \Omega:=\tau(\mathcal{D})$ is then a locally closed smooth surface in $\mathbb{P} T_{X}$ containing $C^{\prime}$. Denote by $\pi: T_{X} \longrightarrow X$ the canonical projection, and write $\pi^{\prime}$ for $\left.\pi\right|_{\Omega}$.

We consider the projective second fundamental form $\sigma$ on VMRTs. Let $[\alpha] \in$ $C^{\prime}, \pi([\alpha]):=x$. Then, $\sigma_{[\alpha]}: S^{2} T_{[\alpha]}\left(\mathcal{C}_{x}\right) \longrightarrow N_{[\alpha]}$, where $N$ denotes the normal bundle of $\mathcal{C}_{x} \cap \Omega$ in $\mathbb{P} T_{x}(X)$, and $N_{[\alpha]}$ denotes the fiber of $N$ at $[\alpha]$. Denote by $T_{\pi}$ resp. $T_{\pi^{\prime}}$ the relative tangent bundles of $\pi: \mathbb{P} T_{X} \longrightarrow X$ resp. $\pi^{\prime}: \Omega \longrightarrow C$; $\pi^{\prime}=\left.\pi\right|_{\Omega}$; and set $N_{\pi^{\prime}}:=\left.T_{\pi}\right|_{\Omega} / T_{\pi^{\prime}}$. Varying $[\alpha] \in C^{\prime}$, we have on $C^{\prime}$ a holomorphic section $\sigma \in \Gamma\left(C^{\prime}, \operatorname{Hom}\left(S^{2} T_{\pi^{\prime}}, N_{\pi^{\prime}}\right)\right)$. We have

Lemma (3.2.1). The projective second fundamental form $\sigma$ is nowhere vanishing on $C^{\prime}$.

Proof. We compute the degrees of the holomorphic line bundles entering into the picture. On the projective space $\mathbb{P} T_{x}(X)$ for a nonzero vector $\alpha \in T_{x}(X)$ we have the isomorphism $T_{[\alpha]}\left(\mathbb{P} T_{x}(X)\right) \cong \pi^{*} T_{x}(X) / \mathbb{C} \alpha$. This isomorphism depends on the choice of the vector $\alpha$, not just $[\alpha]$. The isomorphism $T_{[\alpha]}\left(\mathbb{P} T_{x}(X)\right) \otimes L_{[\alpha]} \cong$ $\pi^{*} T_{x}(X) / L_{[\alpha]}$, where $L_{[\alpha]}=\mathbb{C} \alpha$ is the tautological line at $[\alpha]$, is then well-defined. Varying over $C^{\prime}$, we then have a canonical isomorphism $T_{\pi} \otimes L \cong \pi^{*} T_{x}(X) / L$ over $C^{\prime}$. Since $\left.L\right|_{C^{\prime}} \cong T_{C^{\prime}}$ canonically, and $C$ is a standard rational curve, it follows 
that $\pi^{*} T_{X} \cong \mathcal{O}(2) \oplus \mathcal{O}(1) \oplus \mathcal{O}$, and we have

$$
\left.\left.T_{\pi}\right|_{C^{\prime}} \cong \pi^{*} T_{X}\right|_{C^{\prime}} / T_{C^{\prime}} \otimes T_{C^{\prime}}^{*} \cong(\mathcal{O}(1) \oplus \mathcal{O}) \otimes \mathcal{O}(-2) \cong \mathcal{O}(-1) \oplus \mathcal{O}(-2) .
$$

Since at $[\alpha], T_{[\alpha]}\left(\mathcal{C}_{x}\right) \cong P_{\alpha} / \mathbb{C} \alpha$, where $P_{\alpha} \subset T_{x}(X)$ is generated by vectors belonging to the positive components of the Grothendieck decomposition of $\pi^{*} T_{X}$, we have $\left.T_{\pi^{\prime}}\right|_{C^{\prime}} \cong \mathcal{O}(1) \otimes \mathcal{O}(-2) \cong \mathcal{O}(-1)$ and $\left.N_{\pi^{\prime}}\right|_{C} \cong \mathcal{O} \otimes \mathcal{O}(-2) \cong \mathcal{O}(-2)$. As a consequence, over $C^{\prime}, \operatorname{Hom}\left(S^{2} T_{\pi^{\prime}}, N_{\pi^{\prime}}\right) \cong \operatorname{Hom}(\mathcal{O}(-2), \mathcal{O}(-2)) \cong \mathcal{O}$ is holomorphically trivial. Since at a generic point $[\alpha] \in C^{\prime}, \sigma_{[\alpha]}$ is nonzero, $\sigma$ must be nowhere vanishing on $C^{\prime}$, as desired.

Alternative proof of Theorem 1, Case (2), continued. The fiber of the canonical projection $\lambda: \mathcal{C} \longrightarrow X$ is 1 -dimensional outside a subset $A$ of codimension $\geq 2$ on $X, \lambda=\left.\pi\right|_{\mathcal{C}}$. Thus, at a generic point $y$ of the hypersurface $H \subset X, \mathcal{C}_{y}$ describes a holomorphic family of quadrics in $\mathbb{P} T_{z}(X)$ as $z$ varies over a neighborhood of $y$. The quadric $\mathcal{C}_{z}$ is non-degenerate if and only if at some smooth point $[\gamma]$ of $\mathcal{C}_{z}$, the projective second fundamental form is non-degenerate. We may choose $C$ to intersect $H$ transversally at a generic point of $y$ lying outside $A$, and deform $C$ to get a 2-dimensional family of minimal rational curves, parametrized by a Euclidean ball, intersecting $H$ transversally in a neighborhood of $y$ in $H$. Lemma (3.2.1) then implies that $\mathcal{C}_{z}$ describes a regular family of non-degenerate quadric curves in $\mathbb{P T}_{z}(X)$ as $z$ varies over a neighborhood of $y$. But this contradicts the assumption on $H$. In other words, the exceptional set $E \subset X$ must be of codimension $\geq 2$, and can be taken to be the set $Z$ in the set-up of Proposition (3.1.3). From the latter we conclude that $X$ is biholomorphic to the model Hermitian symmetric space $S=Q^{3}$, the 3-dimensional hyperquadric, as desired.

(3.3). For the proof of the Main Theorem it remains to consider Case (3), where $d=3$. We start with a preliminary discussion on (not necessarily generic) VMRTs on $X$. There is a subvariety $\mathcal{S} \subset \mathcal{U}$ of the universal family $\mathcal{U}$ of codimension $\geq 2$ such that the tangent map $\tau: \mathcal{U} \rightarrow \mathbb{P} T_{X}$ is holomorphic on $\mathcal{U}-\mathcal{S}$. The pull-back $\tau^{*}\left(L^{*}\right)$ of the dual of the tautological line bundle $L$ on $\mathbb{P} T_{X}$ gives a holomorphic line bundle $F$ on $\mathcal{U}-\mathcal{S}$, which can be extended to $\mathcal{U}$ as a holomorphic line bundle (cf. the proof of Corollary (1.3.1)). Writing $\mathcal{F}$ for the corresponding locally free sheaf on $\mathcal{U}$, the direct image $\mu_{*} \mathcal{F}$ then defines a rank-4 holomorphic vector bundle $V_{o}$ on $X$. The universal family $\mathcal{U}$ is canonically embedded into $\mathbb{P} V_{o}$. The (rational) tangent map $\tau$ then induces a rational map $\mathbb{P} V_{o} \rightarrow \mathbb{P} T_{X}$ which lifts to a holomorphic map $h: V_{o} \otimes \mu^{*} J \longrightarrow T_{X}$ for some holomorphic line bundle $J$ on $X$, such that $h$ does not vanish identically on any hypersurface $H \subset X$. In what follows we write $V$ for $V_{o} \otimes \mu^{*} J$, noting that $\mathbb{P} V=\mathbb{P} V_{o}$.

We are going to prove that $X$ is biholomorphically isomorphic to the 5 -dimensional Fano homogeneous contact manifold of the type $G_{2}$, to be denotecd by $K\left(G_{2}\right)$. We start by proving that $X$ must be of dimension $\geq 5$. By Theorem (1.1.1), we may assume that $X$ is of dimension $\geq 4$. To rule out the possibility that $X$ is of dimension 4, again by Theorem (1.1.1) it suffices to prove

Lemma (3.3.1). There does not exist any 4-dimensional Fano manifold of P $i$ card number 1 with nef tangent bundle such that $c_{1}(X)$ is the positive generator of $\operatorname{Pic}(X) \cong \mathbb{Z}$ and such that there exists a minimal rational curve on $X$ of degree 3 .

Proof. For such a Fano manifold $X$ we have $d=3$. Let $E \subset X$ be the smallest subvariety such that $\lambda: \mathcal{C} \longrightarrow X$ is a regular family over $X-E$, and such that at 
$x \in X-E$, the $\operatorname{VMRT} \mathcal{C}_{x} \subset \mathbb{P} T_{x}(X) \cong \mathbb{P}^{3}$ is a nonsingular rational curve of degree 3 , embedded by a complete linear system. We call such a curve a standard cubic curve in $\mathbb{P}^{3}$. Note that fibers of $\lambda: \mathcal{C} \longrightarrow X$ are 1-dimensional outside a subvariety of $X$ of codimension $\geq 2$. We proceed to prove that $E$ does not contain any hypersurface $H$ of $X$. Suppose $H$ exists. Let $C$ be a standard minimal rational curve of degree 3 intersecting $H$ transversally, at points where fibers of $\lambda: \mathcal{C} \longrightarrow X$ are 1dimensional. To avoid clumsy notations assume that the tautological lifting $C^{\prime}$ of $C$ is nonsingular. We compute the projective second fundamental form $\sigma_{[\alpha]}$ at a point $[\alpha] \in \mathcal{C}_{x}$ over $x \in C$, and adopt the notations as in (3.2). We claim that the analogue of Lemma (3.2.1) remains valid in this case, i.e., that $\sigma$ is nowhere vanishing on $C^{\prime}$. We have $\sigma \in \Gamma\left(C^{\prime}, \operatorname{Hom}\left(S^{2} T_{\pi^{\prime}}, N_{\pi^{\prime}}\right)\right)$. In analogy with the proof of Lemma (3.2.1) and using the splitting $\left.\pi^{*} T_{X}\right|_{C^{\prime}} \cong \mathcal{O}(2) \oplus \mathcal{O}(1) \oplus \mathcal{O}^{2}$, we conclude that, over $C^{\prime},\left.T_{\pi^{\prime}}\right|_{C^{\prime}} \cong \mathcal{O}(-1),\left.N_{\pi^{\prime}}\right|_{C^{\prime}} \cong \mathcal{O}(-2)^{2}$ and $\operatorname{Hom}\left(S^{2} T_{\pi^{\prime}}, N_{\pi^{\prime}}\right) \cong \mathcal{O}^{2}$. Since a generic point $x \in C$ lies on $X-E$, so that $\mathcal{C}_{x}$ is isomorphic to the standard cubic curve in $\mathbb{P}^{3}$, the projective second fundamental form $\sigma_{[\alpha]}$ must be non-degenerate at a generic point of $C^{\prime}$. As a consequence, $\sigma$ must be nowhere vanishing on $C^{\prime}$, as claimed.

Now let $\left.Q \subset T_{\pi}\right|_{C^{\prime}}$ be the rank-3 holomorphic vector bundle such that $T_{\pi^{\prime},[\alpha]} \subset$ $Q_{[\alpha]}$ at $[\alpha] \in C^{\prime}$, and the quotient $Q_{[\alpha]} / T_{\pi^{\prime},[\alpha]}$ is the (1-dimensional) image of $\sigma_{[\alpha]}$. Consider now what one might call the projective third fundmental form $\kappa$ on $C^{\prime}$, which at $[\alpha] \in C^{\prime}$ assigns an element in $T_{\pi,[\alpha]} / Q_{[\alpha]}$. Again, as $\mathcal{C}_{x}$ is isomorphic to the standard cubic curve in $\mathbb{P}^{3}, \kappa$ is not identically zero. In analogy to the proof of Lemma (3.2.1) we have $\left.T_{\pi}\right|_{C^{\prime}} \cong \pi^{*} T_{X} / T_{C^{\prime}} \otimes T_{C^{\prime}}^{*} \cong \mathcal{O}(-1) \oplus \mathcal{O}(-2)^{2}$, and that furthermore $\left.Q\right|_{C^{\prime}} \cong \mathcal{O}(-1) \oplus \mathcal{O}(-2)$, so that

$$
\kappa \in \Gamma\left(C^{\prime}, \operatorname{Hom}\left(S^{3} T_{\pi^{\prime}}, T_{\pi} / Q\right)\right) \cong \Gamma\left(C^{\prime}, \operatorname{Hom}(\mathcal{O}(-3), \mathcal{O}(-2))\right) \cong \Gamma\left(C^{\prime}, \mathcal{O}(1)\right) .
$$

As a consequence, the projective third fundamental form must have a single simple zero along $C^{\prime}$ and no other zeros elsewhere.

Since $C$ is of degree 3 and it intersects $H$ transversally, $H \cap C$ must consist of at least 3 distinct points. There must therefore exist a point $y \in H \cap C$ at which $\kappa(y) \neq 0$. We claim that $\mathcal{C}_{y} \subset \mathbb{P} T_{y}(X) \cong \mathbb{P}^{3}$ must be a standard cubic curve. Recall that $h: V \longrightarrow T_{X}$ is the lifting of the tangent map $\tau: \mathcal{U} \rightarrow \mathbb{P} T_{X}$ to a holomorphic vector bundle homomorphism, with the property that $h$ does not vanish identically on any hypersurface of $X$. Then either $h_{y}: V_{y} \cong T_{y}(X)$, or $h_{y}\left(V_{y}\right) \varsubsetneqq T_{y}(X)$ is a vector subspace of codimension 1 (noting that $\sigma_{[\alpha]} \neq 0$ at $[\alpha]=\left[T_{y}(C)\right]$ ). But in the latter case the strict transform $\tau_{y}\left(\mathcal{U}_{y}\right):=\mathcal{C}_{y}^{o}$ is a curve in $\mathbb{P}\left(h\left(V_{y}\right)\right) \cong \mathbb{P}^{2}$, so that $\kappa(y)=0$, contradicting our choice of $y \in H \cap C$. It follows that on some nonempty open subset of $H, \mathcal{C}_{y}$ must be isomorphic to the standard cubic curve in $\mathbb{P}^{3}$, and hence the same is true at a generic point of $H$, contradicting the choice of $H$. In other words, $E \subset X$ must be of codimension $\geq 2$, as claimed. But even then there is a contradiction. In fact, since $E \subset X$ is of codimenson $\geq 2$, there exists a standard minimal rational curve $C$ lying on $X-E$. For any such $C, \kappa$ must vanish somewhere on $C^{\prime}$, contradicting the fact that every $\mathcal{C}_{x}$ is isomorphic to a standard cubic in $\mathbb{P}^{3}$ for $x \in X-E$. In other words, the 4-dimensional Fano manifold $X$ does not exist, and the proof of Lemma (3.3.1) is complete.

We proceed next to determine the dimension of $X$ in Case (3), i.e., for $d=3$. 
Lemma (3.3.2). Let $X$ be a Fano manifold satisifying the hypothesis of the Main Theorem for which $d=3$, in the notations of Proposition (2.2.1). Then, X must be 5-dimensional.

Proof. Let $D$ be the meromorphic distribution on $X$ spanned at generic points by VMRTs. $D$ is holomorphic on $X-E$ for some subvariety $E \subset X$ of codimension $\geq 2$. Since $d=3$, at a generic point $x \in X, \Gamma\left(\mathcal{U}_{x}, \tau_{x}^{*} \mathcal{O}(1)\right) \cong \Gamma\left(\mathbb{P}^{1}, \mathcal{O}(3)\right) \cong \mathbb{C}^{4}$, and $\operatorname{rank}(D) \leq 4$. If $\operatorname{rank}(D) \leq 3$, then $\mathbb{P} D_{x} \cong \mathbb{P}^{1}$ or $\mathbb{P}^{2}$, and $D$ is integrable by Proposition (3.1.2), contradicting Proposition (3.1.1). Thus, $\operatorname{rank}(D)=4$, and the VMRT $\mathcal{C}_{x} \subset \mathbb{P} D_{x} \cong \mathbb{P}^{3}$ at a generic point $x$ must be isomorphic to the standard cubic curve in $\mathbb{P}^{3}$. Denote by $\varphi: \Lambda^{2} D \longrightarrow T_{X} / D$ the Frobenius form. From the proof of Proposition (3.1.2), for any $[\alpha] \in \mathcal{C}_{x}$ and any $\xi \in D_{x}$ such that $\xi \bmod \mathbb{C} \alpha \in T_{[\alpha]} \mathcal{C}_{x}$, we have $\alpha \wedge \xi \in \operatorname{Ker}\left(\varphi_{x}\right)$. From the cubic expansion of $\mathcal{C}_{x}$ it follows readily that $\operatorname{Ker}\left(\varphi_{x}\right) \subset \Lambda^{2} D_{x}$ must at least be 5 -dimensional, while $\Lambda^{2} D_{x} \cong$ $\mathbb{C}^{6}$, so that $\operatorname{Im}\left(\varphi_{x}\right)$ is 0 or 1 -dimensional. If it is zero at a generic point $x$, then $D$ is integrable, contradicting Proposition (3.1.1). Thus, $\operatorname{Im}\left(\varphi_{x}\right)$ is 1-dimensional at a generic point $x$ on $X-E$, and there exists a meromorphic distribution $W$ of rank 5 on $X$ containing $D$ at generic points such that $W_{x} / D_{x}=\operatorname{Im}\left(\varphi_{x}\right)$ at a generic point. Replacing $E$ by some larger subvariety of $X$ of codimension $\geq 2$ if necessary, we may assume that $W$ is also holomorphic on $X-E$.

We are going to prove that $X$ is of dimension 5 by showing that $W$ agrees with the tangent bundle $T_{X}$. By Proposition (3.1.1) it suffices to show that $W$ is integrable. The proof is already contained in $\mathrm{Hw}$ and [HM5]. Since the proof in the present special case is particularly simple, we include here a self-contained proof. Let $S \subset X$ be a subvariety, $S \supset E$, such that $\operatorname{Im}\left(\varphi_{x}\right)$ is 1-dimensional on $X-S$. $S$ may contain irreducible components of codimension 1 on $X$. Denote by $\mathcal{W}$ the sheaf of germs of holomorphic sections of $W$ on $X-E$. The same notational convention will apply to other distributions. $W$ is integrable if and only if $[\mathcal{W}, \mathcal{W}] \subset \mathcal{W}$. Since on $X-S$ we have $\mathcal{W}=[\mathcal{D}, \mathcal{D}]$, it suffices to show that for any local $D$-valued holomorphic vector fields $\eta_{1}, \eta_{2}, \eta_{3}$ and $\eta_{4}$ on an open subset $U \subset X-S$, the holomorphic vector field $\left[\left[\eta_{1}, \eta_{2}\right],\left[\eta_{3}, \eta_{4}\right]\right]$ must again be $W$-valued. By the Jacobi identity it is enough to show that

$$
\left[\left[\eta_{1}, \eta_{2}\right], \eta_{3}\right] \text { is } W \text {-valued for arbitrary } \eta_{1}, \eta_{2} \text { and } \eta_{3} \text {. }
$$

Since $D$ is spanned by VMRTs, we may assume that there exist 4 linearly independent holomorphic sections $\alpha_{1}, \alpha_{2}, \alpha_{3}$ and $\alpha_{4}$ on $U$ such that $\left[\alpha_{i}(x)\right] \in \mathcal{C}_{x}$ for every $x \in U$. Thus it suffices to establish $(\dagger)$ in the case when $\eta_{3}$ is replaced by any one of these $\alpha_{i}$ 's, which we will simply denote by $\alpha$. We may further asume that there is a holomorphic section $\gamma$ of the universal family $\mu: \mathcal{U} \longrightarrow X$ over $U$ such that $[\alpha(x)]=\tau_{x}(\gamma(x))$ for any $x \in U$, and such that furthermore $\rho(\gamma(x)) \in \mathcal{K}$ is a standard minimal rational curve. There is a filtration $\mathbb{C} \alpha \subset P_{\alpha} \subset Z_{\alpha} \subset D$ of $D$ on $U$ which depends on the choice of $\alpha$, defined at $x \in U$ as follows. $P_{\alpha(x)} \subset D_{x}$ is the 2-dimensional vector subspace containing $\mathbb{C} \alpha(x)$ such that $P_{\alpha(x)} \bmod \mathbb{C} \alpha(x) \cong$ $T_{[\alpha(x)]} \mathcal{C}_{x}$ canonically. $Z_{\alpha(x)} \supset P_{\alpha(x)}$ is the 3 -dimensional vector subspace such that $Z_{\alpha(x)} / P_{\alpha(x)}$ is canonically identified with the nonzero image of the projective second fundamental form $\sigma_{[\alpha(x)]}$ of $\mathcal{C}_{x} \subset \mathbb{P} D_{x}$ at $[\alpha(x)]$. Writing $\mathcal{P}$ resp. $\mathcal{Z}$ for the sheaf of germs of holomorphic sections of $P$ resp. $Z$ and using the cubic expansion of the standard cubic curve in $\mathbb{P}^{3}$, it follows readily that $[\mathcal{P}, \mathcal{Z}]+\mathcal{D}=\mathcal{W}$. To prove $(\dagger)$ it is therefore enough to take $\eta_{1}$ to be $P$-valued, to be denoted by $\xi$, and $\eta_{2}$ to 
be $Z$-valued, to be denoted by $\zeta$. But then, by the Jacobi identity,

$$
\left.\left[\left[\eta_{1}, \eta_{2}\right], \eta_{3}\right]=[[\xi, \zeta], \alpha]=[[\alpha, \zeta], \xi]+[[\xi, \alpha], \zeta]\right] .
$$

However, by the proof of Proposition (3.1.2) the two vector fields $[\alpha, \xi]$ and $[\alpha, \zeta]$ are $D$-valued. (The latter statement is implicit in the statement of Proposition (3.1.2).) It follows that $\left[\left[\eta_{1}, \eta_{2}\right], \eta_{3}\right]$ is $W$-valued, as desired. The proof of Lemma (3.2.2) is complete.

Proof of the Main Theorem, continued. We proceed now to prove that any 5dimensional Fano manifold satisfying the hypothesis of the Main Theorem for which $d=3$ must be biholomorphic to the Fano homogeneous contact manifold $K\left(G_{2}\right)$. In the notations of the proof of Lemma (3.2.2), we have a meromorphic distribution $D$ on $X$ of corank 1, regular outside a subvariety $E \subset X$ of codimension $\geq 2$. Let $S \subset X$ be the smallest subvariety containing $E$ such that (i) the Frobenius form $\varphi: \Lambda^{2} D \longrightarrow T_{X} / D$ is non-degenerate on $X-S$, and (ii) $\left.\pi^{\prime}\right|_{X-S}:\left.\mathcal{C}\right|_{X-S} \longrightarrow X-S$ is a regular family of $\mathbb{P}^{1}$, embedded at $x$ as a standard cubic curve in $\mathbb{P} D_{x} \cong \mathbb{P}^{3}$. $A$ priori $S \supset E$ may contain a hypersurface $H$ of $X$. By Proposition (3.1.4), $X$ is biholomorphic to $K\left(G_{2}\right)$, provided that $S \subset X$ is in fact of codimension $\geq 2$. Without loss of generality we may assume that the canonical projection $\lambda: \mathcal{C} \longrightarrow X$ has 1-dimensional fibers over $X-E$, and that for any $y \in X-E$, there is a standard minimal rational curve passing through $y$, so that in particular the strict transform $\mathcal{C}_{y}^{o}$ of $\tau_{y}: \mathcal{U}_{y} \rightarrow \mathbb{P} T_{y}(Y)$ is 1-dimensional.

To prove that $S \supset E$ does not contain any hypersurface we will proceed by contradiction. Let $H \subset S$ be an irreducible hypersurface of $X$. Let $C \subset X$ be a standard minimal rational curve intersecting $H$ transversally at generic points. We will show that at any $y \in H \cap C, \mathcal{C}_{y} \subset \mathbb{P} D_{y} \cong \mathbb{P}^{3}$ is a standard cubic curve, and that furthermore the Frobenius form $\varphi_{y}$ at $y$ is non-degenerate. For the former property it suffices as in the proof of Lemma (3.3.1) to prove that the third fundamental form $\kappa$ is non-degenerate on the tautological lifting $C^{\prime}$. For this purpose we need to determine the splitting type of $\left.D\right|_{C}$, where for notational simplicity we assume again that $C$ is embedded. Now $\left.\left.D\right|_{C} \subset T_{X}\right|_{C} \cong \mathcal{O}(2) \oplus \mathcal{O}(1) \oplus \mathcal{O}^{3}$. We have on $C$ the rank-3 holomorphic vector subbundle $Z \subset D$ defined in analogy to the filtration of $\left.T_{X}\right|_{U}$ in the proof of Lemma (3.2.2), where $Z \cong \mathcal{O}(2) \oplus \mathcal{O}(1) \oplus \mathcal{O}$. (Compare this to the vector bundle $Q$ on $C^{\prime}$ in the proof of Lemma (3.2.1), where $Q=\pi^{\prime *} Z \otimes T_{C^{\prime}}^{*}$.) We have $\left.D\right|_{C} / Z \subset T_{X} / Z \cong \mathcal{O}^{2}$, so that $D_{C} / Z \cong \mathcal{O}(-k)$ for some $k \geq 0$. Now, $\operatorname{Hom}(\mathcal{O}(-k), Z) \cong \mathcal{O}(k+2) \oplus \mathcal{O}(k+1) \oplus \mathcal{O}(k)$ is a semipositive vector bundle over $C \cong \mathbb{P}^{1}$. It follows that $H^{1}(C, \operatorname{Hom}(\mathcal{O}(-k), Z))=0$ and that the short exact sequence $\left.\left.0 \longrightarrow Z \longrightarrow D\right|_{C} \longrightarrow D\right|_{C} / Z \longrightarrow 0$ splits, giving $\left.D\right|_{C} \cong$ $\mathcal{O}(2) \oplus \mathcal{O}(1) \oplus \mathcal{O} \oplus \mathcal{O}(-k)$. The third fundamental form $\kappa$ gives a section over $C^{\prime}$ of $\operatorname{Hom}(\mathcal{O}(-3), \mathcal{O}(-k-2)) \cong \mathcal{O}(1-k)$. Since $\kappa$ is nonzero at a generic point of $C^{\prime}$, we must have $1-k \geq 0$, i.e., $k \leq 1$. On the other hand, if $k=0$, then $\left.\left(T_{X} / D\right)\right|_{C} \cong \mathcal{O}$, so that for the Frobenius form $\varphi$ restricted to $C, \varphi(\alpha, \eta)=0$ whenever $\alpha \in T_{x}(C)$ and $\eta \in D_{x}$. Fixing $x \in X$ and varying the minimal rational curve $C$ passing through $x$, we conclude by polarization that $\varphi_{x}=0$, contradicting the fact that $\varphi$ is non-degenerate at a generic point. We conclude therefore that $\left.D\right|_{C} \cong \mathcal{O}(2) \oplus \mathcal{O}(1) \oplus \mathcal{O} \oplus \mathcal{O}(-1)$. Then, $\kappa$ gives a holomorphic section over $C^{\prime}$ of $\operatorname{Hom}(\mathcal{O}(-3), \mathcal{O}(-3)) \cong \mathcal{O}$. As it is nonzero at a generic point of $C^{\prime}$, it must be nonvanishing everywhere, as claimed. 
It remains to prove that the Frobenius form $\varphi$ is everywhere nondegenerate on $C$. Knowing the splitting type of $\left.D\right|_{C}$, we conclude that $\left.\left(T_{X} / D\right)\right|_{C} \cong \mathcal{O}(1)$. For $x \in C$ we have $\varphi_{x}(\alpha, \zeta)=0$ whenever $\zeta \in Z_{x}$. Thus $\varphi$ induces a homomorphism $\psi:\left.T_{C} \otimes D\right|_{C} /\left.Z \longrightarrow\left(T_{X} / D\right)\right|_{C}$, i.e., $\psi: \mathcal{O}(2) \otimes \mathcal{O}(-1) \longrightarrow \mathcal{O}(1)$. Since $\varphi$ is nondegenerate at a generic point of $C$, so is $\psi$, and $\psi$ must then be an isomorphism on $C^{\prime}$. This implies that $\varphi$ is non-degenerate on $C$, so that $\varphi$ is non-degenerate at a generic point of $H$. This contradicts the minimality of $S$ in its definition. We conclude that $S \subset X$ is of codimension $\geq 2$, and that, as a consequence of Proposition (3.1.4), $X$ is biholomorphically isomorphic to the Fano homogeneous contact manifold $K\left(G_{2}\right)$. The proof of the Main Theorem is complete.

Finally, we note that the 3 cases of rational homogeneous manifolds as listed in the Main Theorem do satisfy the hypothesis given there. Since they are homogeneous their tangent bundles are nef, being spanned by global holomorphic vector fields. The VMRTs are well-known and of dimension 1, pertaining to the cases of $d=1,2,3$. Since they are of the form $G / P$ with $P \subset G$ a maximal parabolic subgroup, the second Betti numbers are equal to 1 . The only part of the hypothesis which is not immediate is the condition $b_{4}(X)=1$. For $X=\mathbb{P}^{2}$, of real dimension $4, b_{4}(X)=1$. For $X=Q^{3}$, of real dimension $6, b_{4}(X)=b_{2}(X)=1$ by Poincaré duality. For $X$ being the Fano homogeneous contact manifold $K\left(G_{2}\right)$, the component $\mathcal{K}$ is itself a rational homogeneous manifold of Picard number 1 and of type $G_{2}$, carrying a rank-2 $G_{2}$-invariant distribution which assigns to each $[C] \in \mathcal{K}$ the 2-dimensional space of holomorphic sections of the positive component of rank 1 of the normal bundle $N_{C \mid X}$ of $C$ in $X$. It follows that $\mathcal{K}$ must be the rational homogeneous manifold of type $G_{2}$ corresponding to the short node, which, as a complex manifold, is biholomorphic to the 5-dimensional hyperquadric $Q^{5}$. From Lemma (1.2.3), $b_{k}(X)=b_{k}\left(Q^{5}\right)$ for any nonnegative integer $k$. But for an odd-dimensional hyperquadric the Betti numbers agree with those of the projective space of the same dimension, so that $b_{4}(X)=b_{4}\left(Q^{5}\right)=1$, as asserted.

\section{ACKNOWLEDGEMENT}

The author would like to thank Jun-Muk Hwang for his insightful remarks which led to substantial simplification of the original proof of the Main Theorem.

\section{REFERENCES}

[Ca] Campana, F.: On twistor spaces of the class $\mathcal{C}$, J. Diff. Geom. 33 (1991), 541-549. MR 92g:32059

[CP1] Campana, F. and Peternell, T.: Projective manifolds whose tangent bundles are numerically effective, Math. Ann. 289 (1991), 169-187. MR 91m:14061

[CP2] Campana, F. and Peternell, T.: 4-folds with numerically effective tangent bundles, Manus. Math. 79 (1993), 225-238. MR 94e:14052

[Fu] Fujita, T.: Classification Theories of Polarized Varieties, London Math. Soc. Lecture Notes Series 155, Cambridge, 1990. MR 93e:14009

[GR] Grauert, H. and Remmert, R.: Coherent Analytic Sheaves, Springer-Verlag, BerlinHeidelberg-New York, 1984. MR 86a:32001

[Ho] Hong, J.-H: Fano manifolds with geometric structures modeled after homogeneous contact manifolds, International J. Math. 11 (2000), 1203-1230. MR 2001m:53128

[Hw] Hwang, J.-M.: Rigidity of homogeneous contact manifolds under Fano deformation, J. Reine Angew. Math. 486 (1997) 153-163. MR 98h:32031

[HM1] Hwang, J.-M. and Mok, N.: Uniruled projective manifolds with irreducible reductive G-structures, J. Reine Angew. Math. 490 (1997), 55-64. MR 99h:32034 
[HM2] Hwang, J.-M. and Mok, N.: Rigidity of irreducible Hermitian symmetric spaces of the compact type under Kähler deformation, Invent. Math 131 (1998), 393-418. MR 99b:32027

[HM3] Hwang, J.-M. and Mok, N.: Varieties of minimal rational tangents on uniruled manifolds, Several Complex Variables, edited by M. Schneider and Y.-T. Siu, MSRI publications 37 Cambridge University Press (2000), pp.351-389. CMP 2000:11

[HM4] Hwang, J.-M. and Mok, N.: Cartan-Fubini type extension of holomorphic maps for Fano manifolds with Picard number 1, J. Math. Pures et Appliquées (9) 80 (2001), 563-575.

[HM5] Hwang, J.-M. and Mok, N.: Deformation rigidity of the rational homogeneous space associated to a long root. Preprint.

[KMM] Kollár, J., Miyaoka, Y., and Mori, S.: Rationally connected varieties, J. Alg. Geom. 1 (1992), 429-448. MR 93i:14014

[Mk] Mok, N.: The uniformization theorem for compact Kähler manifolds of nonnegative holomorphic bisectional curvature, J. Diff. Geom. 27 (1988), 179-214. MR 89d:53115

[Mr] Mori, S.: Projective manifolds with ample tangent bundles, Ann. Math. 110 (1979), 593-606. MR 81j:14010

[Mu Mukai, S.: Biregular classification of Fano 3-folds and Fano manifolds of coindex 3, Proc. Natl. Acad. Sci. U.S.A. 86 (1989), 3000-3002. MR 90g:14024

[Si] Siu, Y.-T.: Lectures on Hermitian-Einstein Metrics for Stable Bundles and on KählerEinstein Metrics, DMV Seminar Band 8, Birkhäuser Verlag, Basel, 1987. MR 89d:32020

[Sp] Spanier, E. H. Algebraic Topology, Tata McGraw-Hill Publishing Co., Bombay-New Delhi, 1966. MR 35:1007

[SY] Siu, Y.-T. and Yau, S.-T.: Compact Kähler manifolds with positive bisectional curvature, Invent. Math. 59 (1980), 189-204. MR 81h:58029

[UY] Uhlenbeck, K. and Yau, S.-T.: On the existence of Hermitian-Yang-Mills connections in stable vector bundles, Comm. Pure Appl. Math. 39 (1986), 257-293. MR 88i:58154

[Zh] Zheng, F.: On semi-positive threefolds. Thesis, Harvard, 1990.

Department of Mathematics, The University of Hong Kong, Pokfulam Road, Hong KONG 